\title{
Simulation of confined magnetohydrodynamic flows using a pseudo-spectral method with volume penalization
}

\author{
Jorge A. Morales ${ }^{1}$, Matthieu Leroy ${ }^{2}$, Wouter J.T. Bos ${ }^{1}$, Kai Schneider ${ }^{2}$ \\ ${ }^{1}$ LMFA-CNRS, École Centrale de Lyon, Université de Lyon, France \\ ${ }_{2}$ M2P2-CNRS, Aix-Marseille Université, Marseille, France
}

\begin{abstract}
A volume penalization approach to simulate magnetohydrodynamic (MHD) flows in confined domains is presented. Here the incompressible visco-resistive MHD equations are solved using parallel pseudo-spectral solvers in Cartesian geometries. The volume penalization technique is an immersed boundary method which is characterized by a high flexibility for the geometry of the considered flow. In the present case, it allows to use other than periodic boundary conditions in a Fourier pseudo-spectral approach. The numerical method is validated and its convergence is assessed for two- and three-dimensional hydrodynamic (HD) and MHD flows, by comparing the numerical results with results from literature and analytical solutions. The test cases considered are two-dimensional Taylor-Couette flow, the $z$-pinch configuration, three dimensional Orszag-Tang flow, ohmic-decay in a periodic cylinder, three-dimensional Taylor-Couette flow with and without axial magnetic field and three-dimensional Hartmann-instabilities in a cylinder with an imposed helical magnetic field.
\end{abstract}

Keywords: MHD, immersed boundary, penalization method, pseudo-spectral, Hartmann-instabilities, helical magnetic field, Taylor-Couette

\section{Introduction}

Magnetohydrodynamics is the discipline that studies the interaction between conducting fluids and magnetic fields. Depending on the topology and the intensity of the magnetic field, as well as on the values of the kinematic viscosity and the magnetic diffusivity, numerous different flow behaviors can be observed. As a function of the viscosity the flows can vary from a laminar to a highly turbulent state and the magnetic diffusivity allows to change the dynamics from a highly diffusive transport to an almost frozen-in advection of the magnetic field. Therefore, in the turbulent state, even in the statistically homogeneous case, a large range of dynamically active scales can be observed. Most of the interesting applications of MHD are however not statistically homogeneous due to the presence of solid walls. Examples are the planetary dynamo mechanism, magnetically confined fusion plasmas and industrial applications involving liquid metals $[1,2]$. In order to study these phenomena, either experiments need to be carried out, or a set of nonlinear differential equations must be solved, combined with adequately chosen boundary conditions. In most cases, these equations cannot be solved analytically, so that numerical integration is needed in order to describe the dynamics. A wide range of MHD solvers have been developed over the last decades and an exhaustive listing is beyond the scope of this paper. Nevertheless we will mention several approaches which are used to compute MHD in wall-bounded geometry. A review of MHD solvers developed to compute fusion-plasma-related flows is given in [3], solvers aiming at a description of dynamo computations are, for example, given in $[4,5,6]$ and computations investigating the magnetorotational instability in bounded domain were reported by Rüdiger and Shalybkov [7], Gissinger et al. [8] and Willis and Barenghi [9]. An early numerical approach to study MHD in cylindrical geometry was proposed and validated by Shan et al. [10] and more recently applied to spherical geometry by Mininni et al. [11]. Most of these solvers are adapted to either a single geometry or a particular application. To change the geometry substantial effort must be invested to adapt the numerical mesh or to change the basis functions used in the numerical algorithm.

We present in this manuscript the implementation and validation of the volume-penalization method applied to magnetohydrodynamics. The strength of this approach is the high flexibility in the geometry and the ease of implementation. The volume penalization method is an immersed boundary method [12], in which both the fluid region and 
the confining boundaries are part of the same computational domain. The influence of the boundaries is then modeled by adding a force or drag term to the dynamical equations in the part of the domain in which the boundaries are to be present. In the volume-penalization method, for the hydrodynamic case, the solid bodies are modeled as porous media whose permeability tends to zero. This so called Navier-Stokes/Brinkman model, where the penalization source term in the momentum equation corresponds to the Darcy drag, was first proposed by Arquis and Caltagirone [13] in the context of the natural convection flow inside a cavity with porous walls. It was then generalized to study fluid - porous wall - solid boundary systems [14]. In addition to being physically motivated, this model is mathematically justified, since Angot et al. [15] rigorously proved that the method converges to the Navier-Stokes equations combined with no-slip boundaries, when the porosity in the part of the domain corresponding to the boundaries is taken infinitesimally small. A first use of the method in combination with a pseudo-spectral Navier-Stokes solver was reported in [16]. An extensive validation of the method for three dimensional fixed and moving boundaries is reported in [17]. The strength of the combination of a pseudo-spectral Navier-Stokes solver with the penalization method is the compromise between accuracy and ease of implementation while retaining a great flexibility in the choice of the geometry of the boundaries. We have already used this method to study two-dimensional MHD [18, 19, 20] which allowed to compare square, circular and periodic boundaries using the same Cartesian grid and numerical method. In the present paper we will present a detailed validation of the method for two and three-dimensional confined HD and MHD flows.

The manuscript is organized as follows. In section $2-3$, we expose the physical model and its numerical discretization. As a first validation, section 4 presents two-dimensional kinematic and magnetic test cases together with a comparison to analytical results. Validation of the three-dimensional periodic MHD calculations is exposed in section 5.1. Subsequently ohmic decay is considered in section 5.2. Sections 5.3 and 5.4 compare our three-dimensional results for 3D Taylor-Couette HD and MHD flows with those available in the literature, as further validation. Section 5.5 reports on the flow induced in a conducting fluid by the presence of an imposed helical magnetic field.

\section{MHD Equations}

The media we study are isothermal, incompressible and we consider constant permeability $\mu$, permittivity $\varepsilon$ and conductivity $\sigma$ of the material. The MHD equations for this case are the Navier-Stokes equation (including the Lorentz force) and the induction equation (that combines Ohm's law, Faraday's equation and Ampère's law). Introducing conventional normalization of the velocity by the Alfvén velocity $C_{a}=B_{0} / \sqrt{\rho \mu}$, a reference magnetic field $B_{0}$ and a conveniently chosen lengthscale $L$, the normalized equations read,

$$
\frac{\partial \boldsymbol{u}}{\partial t}-v \nabla^{2} \boldsymbol{u}=-\nabla \Pi+\boldsymbol{u} \times \boldsymbol{\omega}+\boldsymbol{j} \times \boldsymbol{B}
$$

and

$$
\frac{\partial \boldsymbol{B}}{\partial t}-\lambda \nabla^{2} \boldsymbol{B}=\nabla \times[\boldsymbol{u} \times \boldsymbol{B}],
$$

where $v$ is the kinematic viscosity, $\lambda$ the magnetic diffusivity $\left(\lambda=(\sigma \mu)^{-1}\right)$ and $\rho=1$ is the density. The vorticity $\omega$ and current density $\boldsymbol{j}$ are given by

$$
\begin{aligned}
\boldsymbol{\omega} & =\nabla \times \boldsymbol{u} \\
\boldsymbol{j} & =\nabla \times \boldsymbol{B},
\end{aligned}
$$

and $\Pi=P+\frac{1}{2} \boldsymbol{u}^{2}$ is the modified pressure. The velocity field $\boldsymbol{u}$ is considered incompressible and the magnetic field $\boldsymbol{B}$ divergence free,

$$
\begin{aligned}
\nabla \cdot \boldsymbol{u} & =0, \\
\nabla \cdot \boldsymbol{B} & =0 .
\end{aligned}
$$

To complete the problem one needs to specify the initial and the boundary conditions corresponding to the physical system that we are interested in. In particular the boundary conditions will be discussed in more detail in the rest of this manuscript. 


\section{The numerical code}

The penalization method was introduced into two independently developed pseudo-spectral MHD solvers, the L-code from Lyon and the M-code from Marseille. The cross-check of the results obtained by the two codes allowed a careful debugging and implementation of the method.

\subsection{Pseudo-spectral discretization}

A classical Fourier pseudo-spectral method is used for the spatial discretization of a cubic periodic domain $\Omega$ of size $2 \pi$ [21]. The physical size of the domain can be modified rescaling the box by multiplying by $L_{x}, L_{y}$ and $L_{z}$. Spatial derivatives are evaluated in Fourier space and multiplications are computed in physical space. In the following we denote the Fourier Transform by the symbol ${ }^{\wedge}$ or $\mathcal{F}\{\}$. All fields are represented as truncated Fourier series and here we show this representation explicitly for the velocity:

$$
\begin{aligned}
& \boldsymbol{u}(\boldsymbol{x}, t)=\sum_{k_{x}=-N_{x} / 2}^{N_{x} / 2-1} \sum_{k_{y}=-N_{y} / 2}^{N_{y} / 2-1} \sum_{k_{z}=-N_{z} / 2}^{N_{z} / 2-1} \widehat{\boldsymbol{u}}(\boldsymbol{k}, t) e^{i \boldsymbol{k} \cdot \boldsymbol{x}}, \\
& \widehat{\boldsymbol{u}}(\boldsymbol{k}, t)=\frac{8 \pi^{3}}{N_{x} N_{y} N_{z}} \sum_{n_{x}=0}^{N_{x}-1} \sum_{n_{y}=0}^{N_{y}-1} \sum_{n_{z}=0}^{N_{z}-1} \boldsymbol{u}\left(\boldsymbol{x}_{\mathbf{n}}, t\right) e^{-i \boldsymbol{k} \cdot \boldsymbol{x}_{\mathbf{n}}},
\end{aligned}
$$

with the wave vector $\boldsymbol{k}=\left(k_{x}, k_{y}, k_{z}\right)$ where $-N_{x} / 2 \leq k_{x} \leq N_{x} / 2-1,-N_{y} / 2 \leq k_{y} \leq N_{y} / 2-1,-N_{z} / 2 \leq k_{z} \leq N_{z} / 2-1$, $\boldsymbol{x}_{\mathbf{n}}=\left(n_{x} 2 \pi / N_{x}, n_{y} 2 \pi / N_{y}, n_{z} 2 \pi / N_{z}\right) \in[0,2 \pi]^{3}$ with $n_{x}=0, \ldots, N_{x}-1, n_{y}=0, \ldots, N_{y}-1, n_{z}=0, \ldots, N_{z}-1$. The number of grid points in $x, y$ and $z$-direction, $N_{x}, N_{y}$ and $N_{z}$, respectively, can be adapted to obtain the accuracy needed in the different directions. To avoid aliasing errors, i.e., the production of small scales due to nonlinear terms which are not resolved on the grid, the velocity and magnetic fields are dealiased at each time step by truncating its Fourier coefficients using the $2 / 3$ rule [21]. For the transformation between physical and Fourier space two different Fourier transforms were used in the two codes, firstly the P3DFFT routine, based on the FFTW library, secondly the JMFFT library. Both Fourier Transforms have an order of complexity of $N \log _{2} N$ with $N=N_{x} N_{y} N_{z}$.

The pressure term can be eliminated from the equations in spectral space by using the incompressibility condition of the medium. This introduces the projector $P_{i j}=\delta_{i j}-k_{i} k_{j} / k^{2}$ in front of the nonlinear term. The Eqs. (1) and (2) in spectral space are then written:

$$
\begin{aligned}
\frac{\partial \hat{u}_{i}}{\partial t}+v k^{2} \hat{u}_{i} & =P_{i j}\left\{(\widehat{\boldsymbol{u} \times \boldsymbol{\omega}}+\widehat{\boldsymbol{j} \times \boldsymbol{B}})_{j}\right\}, \\
\frac{\partial \hat{B}_{i}}{\partial t}+\lambda k^{2} \hat{B}_{i} & =[i \boldsymbol{k} \times(\widehat{\boldsymbol{u} \times \boldsymbol{B}})]_{i},
\end{aligned}
$$

where $k^{2}=\left|\boldsymbol{k}^{2}\right|$.

\subsection{Penalization method}

The volume penalization method is based on the idea of modeling solid bodies as porous media whose permeability tends to zero. The flow is considered in a domain in which both fluid and solid domains are embedded. The difference between the fluid and solid subdomain is the permeability. In the fluid domain the permeability is infinite and in the solid domain the permeability tends to zero. The method allows to consider an arbitrary shape and number of obstacles. The equations are modified by adding the penalization term:

$$
\begin{aligned}
\frac{\partial \boldsymbol{u}}{\partial t} & =\boldsymbol{u} \times \boldsymbol{\omega}-\nabla \Pi+\nu \nabla^{2} \boldsymbol{u}+\boldsymbol{j} \times \boldsymbol{B}-\frac{\chi}{\eta}\left(\boldsymbol{u}-\boldsymbol{u}_{\text {wall }}\right) \\
\frac{\partial \boldsymbol{B}}{\partial t} & =\nabla \times(\boldsymbol{u} \times \boldsymbol{B})+\lambda \nabla^{2} \boldsymbol{B}-\frac{\chi}{\eta}\left(\boldsymbol{B}-\boldsymbol{B}_{\text {wall }}\right),
\end{aligned}
$$


with $\boldsymbol{u}_{\text {wall }}$ and $\boldsymbol{B}_{\text {wall }}$ the imposed values of the velocity and magnetic field in the solid domain and $\eta$ is the permeability of the solid domain, i.e., the penalization parameter, which could be different for each equation, and $\chi(\boldsymbol{x}, t)$ the mask function (see Fig. 1):

$$
\chi(x, t)= \begin{cases}0 & \text { for } x \in \Omega_{f}, \text { the fluid domain } \\ 1 & \text { for } x \in \Omega_{s}, \text { the solid domain. }\end{cases}
$$

According to Eqs. (11) and (12), the flow is governed by the Navier-Stokes and induction equations in $\Omega_{f}$, and by Darcy's law in $\Omega_{s}$ for small $\eta$ (for more details see Appendix A). As mentioned in the introduction, the convergence of the velocity of the penalized equation in the limit of vanishing $\eta$ to the solution of the velocity of the NavierStokes equations with no-slip boundary conditions was rigorously proved by Angot et al. [15] for fixed obstacles. The estimates were then refined by Carbou and Fabrie [22], who demonstrated that the solution of the penalized equations converges in the $L_{2}$-norm with $\sqrt{\eta}$ towards the solution of the non-penalized equations with Dirichlet boundary conditions. Similar results are anticipated for the induction equation.

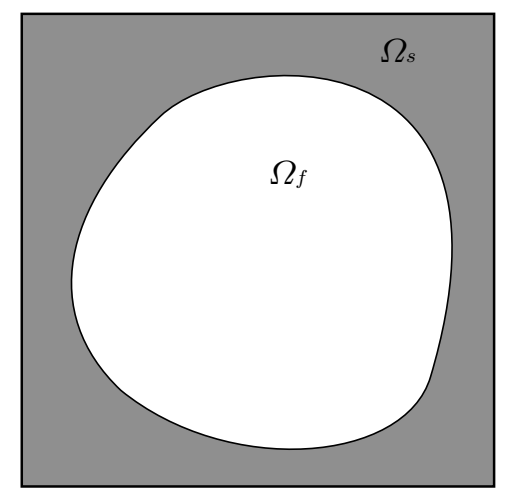

Figure 1: The computational domain $\Omega$ contains both the fluid domain $\Omega_{f}$ and the solid domain $\Omega_{s}$.

To use a pseudo-spectral solver we need to Fourier-transform Eqs. (11) and (12) and we obtain

$$
\frac{\partial \hat{u}_{i}}{\partial t}+v k^{2} \hat{u}_{i}=P_{i j}\left\{\left[\widehat{\boldsymbol{u} \times \boldsymbol{\omega}}+\widehat{\boldsymbol{j} \times \boldsymbol{B}}-\mathcal{F}\left\{\frac{\chi}{\eta}\left(\boldsymbol{u}-\boldsymbol{u}_{\text {wall }}\right)\right\}\right]_{j}\right\} .
$$

We stress here that the Riesz projector $P_{i j}$ is also applied to the penalization term. Indeed, this form straightforwardly appears in the Fourier-transformed equations when the pressure is eliminated by solving a Laplace equation. The fact that the projector also acts on the penalization term is important to ensure incompressibility, since the penalization term is not necessarily divergence free at the fluid-solid boundary. This is also the case for the magnetic field. Due to the penalization term, the magnetic field is no longer divergence free. One way used in the L-code to cure this is to add an auxiliary pressure to the magnetic field

$$
\frac{\partial \boldsymbol{B}}{\partial t}-\lambda \nabla^{2} \boldsymbol{B}=\nabla \times[\boldsymbol{u} \times \boldsymbol{B}] \underbrace{-\nabla \Xi}_{\begin{array}{c}
\text { Auxiliary } \\
\text { pressure }
\end{array}} \underbrace{-\frac{\chi}{\eta}\left(\boldsymbol{B}-\boldsymbol{B}_{\text {wall }}\right)}_{\text {Penalization term }} .
$$

In the absence of boundaries in the domain, this pressure gradient would be equal to zero, as can be directly seen by solving a Laplace equation for $\Xi$ and using the solenoidality constraint, Eq. (6). Indeed the (curl)-term $i \boldsymbol{k} \times(\widehat{\boldsymbol{u} \times \boldsymbol{B}})$ is necessarily divergence free. Eliminating the pressure from Eq. (15), we find for the Fourier-transformed equation for the magnetic field,

$$
\left.\frac{\partial \hat{B}_{i}}{\partial t}+\lambda k^{2} \hat{B}_{i}=P_{i j}\left\{[i \boldsymbol{k} \times \widehat{(\boldsymbol{u} \times \boldsymbol{B}})-\mathcal{F}\left\{\frac{\chi}{\eta}\left(\boldsymbol{B}-\boldsymbol{B}_{\text {wall }}\right)\right\}\right]_{j}\right\},
$$


which guarantees the incompressibility.

Another way adopted in the M-code to impose the constraint $\nabla \cdot \boldsymbol{B}=0$ is the so-called Helmholtz decomposition of a vector field, i.e, a decomposition into its rotational and irrotational part $\boldsymbol{B}=\nabla \times \boldsymbol{\Psi}+\nabla \Phi$. Solving Eq. (12) we obtain a field $\boldsymbol{B}_{n+1}^{*}$ which is not divergence free because of the contribution from the penalization term. We take the divergence of $\boldsymbol{B}_{n+1}^{*}$ to eliminate the irrotational part and we get $\nabla \cdot \boldsymbol{B}=\nabla^{2} \Phi$. We solve the Poisson equation to evaluate $\Phi$ and to calculate its gradient that we substract from $\boldsymbol{B}_{n+1}^{*}$. Hence we have the new divergence free magnetic field $\boldsymbol{B}_{n+1}$ with $\nabla \cdot \boldsymbol{B}_{n+1}=\nabla \cdot\left(\boldsymbol{B}_{n+1}^{*}-\nabla \Phi\right)=0$.

The penalization method allows for a simple implementation of complex geometries, since to change the shape of the walls, one only needs to redefine the mask function. This is a huge advantage, because almost no effort is required to modify the shape of the flow geometry during an investigation and arbitrarily complex shapes can be considered. Several limitations should however be mentioned. First, the dynamical equations are solved in both the fluid domain and the penalized domain, so that, if the penalized domain is large, an important part of the numerical resources is used to compute the dynamically unimportant flow inside the walls (see for example section 5.4). Second, here no mesh refinement near the wall is used, so that, to capture small scale dynamics near boundaries, one needs to globally increase the resolution. Third, the boundary conditions imposed by the numerical method need to be satisfied in the computational domain. Let us explain this latter point in more detail.

Intrinsically, the boundary conditions of the Fourier pseudo-spectral solver are periodic in the three directions. Thus in the computational domain this periodicity must be satisfied. This imposes certain constraints on the geometries and especially on the boundary conditions that can be considered. If in the geometry sketched in Fig. 1 the solid domain corresponds to no-slip walls, i.e. $\boldsymbol{u}_{\text {wall }}=0$, the periodicity condition is met automatically. However, if the outer-walls are chosen to move in solid-body rotation anti-clockwise, the left border of the domain will move downwards whereas the right border will move upwards. In that case the periodicity condition is not satisfied. A solution to this problem is to add a third, unpenalized, subdomain outside the walls which will allow to respect the periodic boundary conditions of the computational domain. This solution is sketched in Fig. 2 (left). However it has an inconvenience which we will describe below.

Discontinuities in the velocity field, or in its gradients, are a source of Gibbs oscillations. These oscillations are an unavoidable feature in the present approach, and as long as their amplitude is small compared to physical effects, they do not constitute a serious problem in most cases. When discontinuities become strong, the Gibbs-oscillations also increase in size. Considering Fig. 2 (left), it is clear that if the solid domain turns and the outer fluid domain is very small, the velocity gradient becomes strong in the outer fluid domain and the velocity gradient will be almost discontinuous on the interface between $\Omega_{s}$ and the outer $\Omega_{f}$. Gibbs oscillations might become strong in this case. One solution is the following. Instead of imposing in $\Omega_{s}$ a solid body rotation, we impose a velocity profile which gives the correct boundary condition at the solid-fluid interface, and which smoothly tends to zero towards the edges of the computational domain Fig. 2 (right). The latter solution is slightly more complicated since an interpolation needs to be computed, using a Hermite's interpolating polynomial for instance. Its advantage is that the continuity of the solution and its derivatives between the boundary value and zero value can be imposed in a smooth way, which yields an improved order of convergence of the algorithm, as we will see in section 4.1 and 4.2.

Another drawback of the penalization method is that it is not yet possible to impose inhomogeneous Neumann conditions at the boundaries. It is therefore not yet possible to impose arbitrary values of the velocity gradient or magnetic gradient, for example, to impose the vorticity and the current density at the walls. This would in particular be important to model the influence of solid boundaries with arbitrary magnetic properties on the magnetic field generated in the fluid. A recent investigation by Kadoch et al. [23] presents a technique for implementing homogeneous Neumann conditions using the penalization method. The extension to inhomogeneous Neumann conditions is an important perspective for further research.

\subsection{Time-discretization}

Two different implementations of the penalization method in the time-advancing scheme will be compared. The first is explicit and constrains the penalization parameter $\eta$ to be bigger than the time step $\Delta t$ to avoid numerical instabilities. The second is a semi-implicit implementation that allows the penalization parameter to be independent of the time step. Second and third order time schemes are used. In all approaches an exact integration of the viscous and magnetic diffusion term is used. In the following two sections these different methods are detailed. 

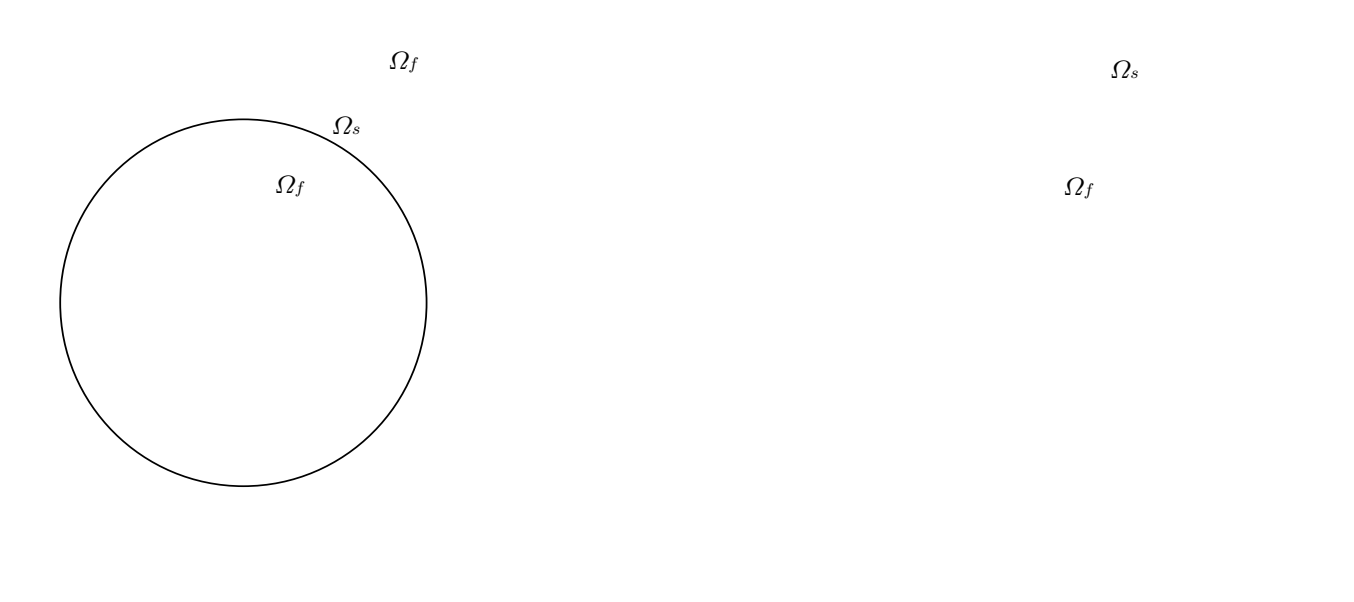

Figure 2: Leaving a part of the domain unpenalized at the edges of the computational domain allows to use inhomogeneous Dirichlet conditions at the fluid solid interface, without violating the periodicity condition at the edge of the domain (left). Another option is to interpolate the imposed velocity to a zero value with a horizontal tangent at the domain frontier with an interpolating Hermite polynomial. The velocity field in the whole computational region belongs then to the $C^{1}$ class (right).

\subsubsection{Explicit implementation of the penalization term}

In this section we detail the time integration of the equations using an explicit treatment of the nonlinear and penalization terms. It must be noted that along with its simplicity and robustness this approach has a drawback: the explicit treatment of the penalization term imposes a stability condition, in addition to the usual CFL condition. An analytical analysis of the magnetic part of the method has yet to be done to check if it adds another stability condition. Up to now no problem occurred if the same stability criterions were used for the velocity and the magnetic field.

The basic time-stepping schemes that are implemented are an adaptive second and third order Adams-Bashforth method (denoted by $\mathrm{AB} 2$ and $\mathrm{AB} 3$ respectively). The use of these schemes fits well into our general concept of compromise between the ease of implementation and computational efficiency. Exact integration of the diffusion term is feasible because the Laplace operator is diagonal in Fourier space and hence no linear system has to be solved. It improves stability of the scheme, avoiding the stability condition $\Delta t<\Delta x^{2} / v$. The remaining terms are discretized explicitly to avoid the solution of nonlinear equations, however it implies a CFL condition on the time step size $\Delta t$ and also a condition due to the explicit discretization of the penalization term, i.e., $\Delta t<\eta$ for $\mathrm{AB} 2$ and $\Delta t<\frac{6}{11} \eta$ for AB3, as linear stability analysis shows [17].

For illustration, the equations will be given for the case of the velocity only. The discretization of the magnetic field is handled analogously, the only difference is the exact form of the nonlinear and penalization terms. First the Navier-Stokes equation is rewritten in the form of a nonlinear evolution equation and transformed into Fourier space,

$$
\begin{aligned}
\partial_{t} \boldsymbol{u}-v \nabla^{2} \boldsymbol{u} & =N(\boldsymbol{u}) \\
\partial_{t} \widehat{\boldsymbol{u}}+v k^{2} \widehat{\boldsymbol{u}} & =\widehat{N}(\widehat{\boldsymbol{u}}) .
\end{aligned}
$$

For the initial condition $\widehat{\boldsymbol{u}}\left(\boldsymbol{k}, t_{n}\right)$, the above equation has the following solution

$$
\widehat{\boldsymbol{u}}\left(\boldsymbol{k}, t_{n+1}\right)=e^{-v \Delta t_{n+1} k^{2}} \widehat{\boldsymbol{u}}\left(\boldsymbol{k}, t_{n}\right)+\int_{t_{n}}^{t_{n+1}} e^{-v\left(t_{n+1}-s\right) k^{2}} \widehat{N}(\widehat{\boldsymbol{u}}(\boldsymbol{k}, s)) d s,
$$

which can be discretized

$$
\begin{aligned}
& \mathrm{AB} 2 \quad \longrightarrow \quad \widehat{\boldsymbol{u}}\left(\boldsymbol{k}, \mathrm{t}_{\mathrm{n}+1}\right)=e^{-v \Delta t_{n+1} k^{2}}\left(\widehat{\boldsymbol{u}}\left(\boldsymbol{k}, t_{n}\right)+\beta_{10} \widehat{N}^{n}+\beta_{11} e^{-v \Delta t_{n} k^{2}} \widehat{N}^{n-1}\right) \\
& \mathrm{AB} 3 \quad \longrightarrow \quad \widehat{\boldsymbol{u}}\left(\boldsymbol{k}, \mathrm{t}_{\mathrm{n}+1}\right)=e^{-v \Delta t_{n+1} k^{2}}\left(\widehat{\boldsymbol{u}}\left(\boldsymbol{k}, t_{n}\right)+\beta_{20} \widehat{N}^{n}+e^{-v \Delta t_{n} k^{2}}\left(\beta_{21} \widehat{N}^{n-1}+\beta_{22} e^{-v \Delta t_{n-1} k^{2}} \widehat{N}^{n-2}\right)\right),
\end{aligned}
$$


with $\widehat{N}^{n}$ denoting the value of the nonlinear term at the time instant $t_{n}$, the second order Adams-Bashforth coefficients

$$
\begin{aligned}
& \beta_{10}=\frac{1}{2} \frac{\Delta t_{n+1}}{\Delta t_{n}}\left(\Delta t_{n+1}+2 \Delta t_{n}\right) \\
& \beta_{11}=-\frac{1}{2} \frac{\Delta t_{n+1}^{2}}{\Delta t_{n}},
\end{aligned}
$$

and the third order Adams-Bashforth coefficients

$$
\begin{aligned}
& \beta_{20}=\frac{\Delta t_{n+1}\left(2 \Delta t_{n+1}^{2}+6 \Delta t_{n} \Delta t_{n+1}+3 \Delta t_{n-1} \Delta t_{n+1}+6 \Delta t_{n}^{2}+6 \Delta t_{n-1} \Delta t_{n}\right)}{6 \Delta t_{n}\left(\Delta t_{n}+\Delta t_{n-1}\right)} \\
& \beta_{21}=\frac{-\Delta t_{n+1}^{2}\left(2 \Delta t_{n+1}+3 \Delta t_{n}+3 \Delta t_{n-1}\right)}{6 \Delta t_{n-1} \Delta t_{n}} \\
& \beta_{22}=\frac{\Delta t_{n+1}^{2}\left(2 \Delta t_{n+1}+3 \Delta t_{n}\right)}{6 \Delta t_{n-1}\left(\Delta t_{n}+\Delta t_{n-1}\right)}
\end{aligned}
$$

where $\Delta t_{n}=t_{n}-t_{n-1}$ [16]. For start-up a first order scheme is used, as two time steps are required to start a secondorder scheme. Similarly a first order and a second order scheme are used to start the third order scheme.

The time step size control is based on the CFL stability limit of the explicit discretization of the nonlinear term, with addition of the stability criterion due to the penalization. Therefore, at each time step $t_{n}$, the maximal point-wise velocity is computed and the new time step is given by $\Delta t_{n+1}=C \Delta x / U_{\max }$ where $C<1$ is the CFL constant and $\Delta x$ is the minimal spatial grid size. Moreover, the time step has to verify the condition $\Delta t_{n+1}<\eta$ (AB2) or $\Delta t_{n+1}<\frac{6}{11} \eta$ (AB3) due to the presence of the penalization term. The same method is applied to the magnetic field and the time step is chosen to be small enough to verify the stability criteria of both the magnetic field and velocity field discretization.

\subsubsection{Semi-implicit implementation}

As noted in the previous section, the stability condition for a third order time scheme constrains $\Delta t<\frac{6}{11} \eta$. To avoid this limitation, an implicit implementation was introduced by Kolomenskiy and Schneider [17] for Burgers' equation and extended to Navier-Stokes' equation by Jause-Labert et al. [24]. In this case the penalization term is evaluated at the time step $t_{n+1}$. The penalization is thus no longer treated together with the nonlinear term. The diffusion term, as in the explicit method, is exactly integrated.

This method is more time-expensive because two additional Fourier transforms are required. In addition to the projection of the nonlinear term, a second projection is realized (that includes the penalization term at $t_{n+1}$ ) to ensure the solenoidal nature of the two considered fields. The fact that the time step can be adaptive (taking into account the CFL condition) makes this technique more suitable for unsteady simulations. The penalization term is introduced using a first order time scheme, which does not influence negatively the precision as long as boundaries are fixed.

The magnetic equations being handled analogously we present the new time scheme for the velocity field

$$
\hat{u}_{i}\left(\boldsymbol{k}, t_{n+1}\right)=P_{i j}\left\{\mathcal{F}\left[\frac{\mathcal{F}^{-1}\left\{Q_{i}^{n}\right\}+\frac{\Delta t}{\eta} \chi u_{\text {wall }_{i}}\left(\boldsymbol{x}, t_{n+1}\right)}{1+\frac{\Delta t}{\eta} \chi}\right]_{j}\right\} .
$$

The third order Adam-Bashforth scheme is retained for the nonlinear terms in this formulation

$$
Q_{i}^{n}=e^{-v \Delta t_{n+1} k^{2}}\left(\widehat{\boldsymbol{u}}\left(\boldsymbol{k}, t_{n}\right)+\beta_{20} \widehat{N}^{n}+e^{-v \Delta t_{n} k^{2}}\left(\beta_{21} \widehat{N}^{n-1}+\beta_{22} e^{-v \Delta t_{n-1} k^{2}} \widehat{N}^{n-2}\right)\right) .
$$

This numerical scheme for a penalization parameter $\eta$ sufficiently small $(\eta<<\Delta t)$ converges towards an explicit modified scheme where the time step $\Delta t$ replaces the penalization parameter $\eta$ and where the nonlinear term vanishes in the solid region. We therefore call this method semi-implicit. This is further explained in Appendix B. For this case with very small penalization parameter, the permeability of the solid media is given by the value of the time step. The asymptotic convergence of the porous boundaries towards a solid wall, if $\eta$ is sufficiently small, is in that case limited by the value $\eta_{\text {effective }} \approx \Delta t$. 


\subsection{Parallel performances}

Both numerical codes are parallelized using MPI libraries. The parallelization performances of the L-code are evaluated on the calculator Vargas of the French high performance computing center IDRIS. The performances are estimated for a HD and MHD calculation for three different grid resolutions. The test cases used are a three-dimensional Taylor-Couette flow for the HD calculation (see section 5.3) and a MHD Taylor-Couette for the MHD calculation (see section 5.4). The comparison is made to ensure that the extra MPI exchanges and cache memory effects of the MHD calculations do not produce important slowdowns.

We find that the MHD calculation is roughly twice as long as the HD one. This is expected as we use for a MHD run the double number of fast Fourier transforms at each iteration. Note that per iteration, using a semi-implicit implementation of the penalization term, we need 12 Fourier transforms for each vector field. The results are shown in table 1 and in Fig. 3.

\begin{tabular}{cc|cccccc}
\hline Processors & & 4 & 8 & 16 & 32 & 64 & 128 \\
\hline $\begin{array}{c}\text { Time per iteration per proc } \\
\text { resolution } 128^{3}(\mathrm{~s})\end{array}$ & Hydro & 1.06 & 0.61 & 0.47 & 0.35 & - & - \\
\hline Time per iteration per proc & Hydro & 10.01 & 5.11 & 2.97 & 1.80 & 1.21 & - \\
resolution $256^{3}(\mathrm{~s})$ & MHD & 19.96 & 10.06 & 6.05 & 3.45 & 2.41 & - \\
\hline Time per iteration per proc & Hydro & - & - & - & 18.61 & 11.68 & 8.20 \\
resolution $512^{3}(\mathrm{~s})$ & MHD & - & - & - & 37.11 & 22.53 & 15.10 \\
\hline
\end{tabular}

Table 1: Parallel performances of the numerical code.

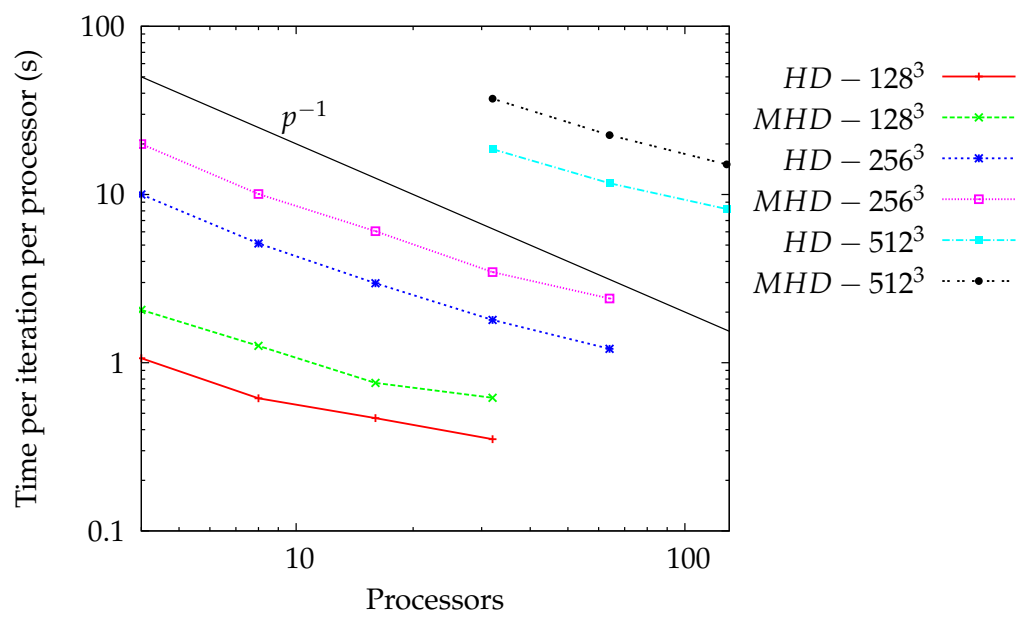

Figure 3: Code scaling in the calculator Vargas of IDRIS for HD and MHD calculations.

The time per iteration per processor scales with the number of processors following an approximate power-law dependence close to the ideal scaling law, 1/(number of processors). The same tests for the M-code, performed on a local cluster, gave a similar scaling behavior. This code requires 9 Fourier transforms per iteration.

\section{Two-dimensional validation}

In this section we present a purely HD test-case, the two-dimensional Taylor-Couette flow and a purely magnetic test-case, the $z$-pinch configuration. For both these cases analytical solutions are known, which allows a careful convergence study and which allows to check different ways to introduce the boundary conditions. 


\subsection{Two-dimensional Taylor-Couette flow}

We consider the classical two-dimensional HD problem of a flow between two coaxial rotating cylinders (e.g., Taylor [25]). The inner cylinder rotates at constant speed, while the outer cylinder is kept at rest. The steady flow solution of the problem is

$$
U_{\theta}(r)=\frac{\Omega_{2} R_{2}^{2}-\Omega_{1} R_{1}^{2}}{R_{2}^{2}-R_{1}^{2}} r+\frac{\left(\Omega_{1}-\Omega_{2}\right) R_{1}^{2} R_{2}^{2}}{R_{2}^{2}-R_{1}^{2}} \frac{1}{r},
$$

where $\Omega_{(1,2)}$ are the angular velocities of the cylinders, $R_{(1,2)}$ the radii of the cylinders and $r$ the cylindrical coordinate (see Fig. 4).

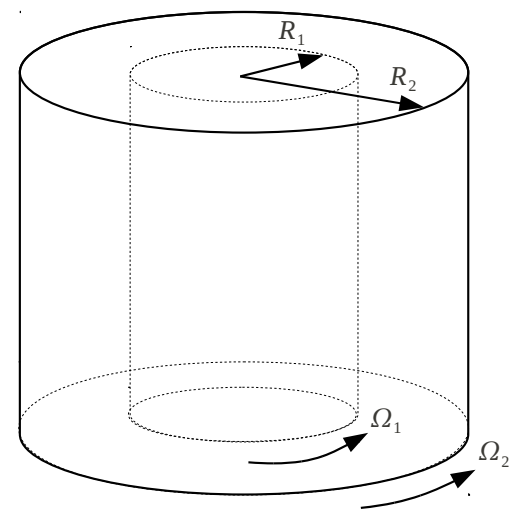

Figure 4: Taylor-Couette flow configuration.

The relative $L_{2}$ error in the fluid domain $\left\|f_{\text {numerical }}-f_{\text {analytical }}\right\|_{L_{2}} /\left\|f_{\text {analytical }}\right\|_{L_{2}}$ with $f$ being the considered field, is calculated for different penalization parameters $\eta$ and number of grid points $N$, in one direction with $N=N_{x}=N_{y}$.

As mentioned above, the present calculation is entirely HD. The simulations are carried out until a steady state is obtained, so that the error is independent of the time discretization. A cubic domain with size-length $2 \pi$ is considered, the time step is fixed to a value $\Delta t=5.10^{-5}$ and the kinematic viscosity $v=1$. The radii $R_{1}, R_{2}$ are $(0.32 \pi, 0.82 \pi)$ respectively. At $t=0$ the fluid domain is at rest and the inner-cylinder is set into movement with a fixed velocity $U_{\theta}\left(R_{1}\right)=1$ while the velocity $U_{\theta}\left(R_{2}\right)$ is kept equal to zero. The runs are stopped when the time $t_{\max }=5$ is reached. At this time instant, the difference in the kinetic energy between two iterations is less than $10^{-9}$ (for a kinetic energy of order unity), which indicates that a steady state is satisfactorily achieved.

The velocity profile imposed in the mask is chosen in two different ways, corresponding to the discussion in section 3.2. In the first case, the velocity in the inner cylinder is straightforwardly set to a solid-body rotation, $U_{\theta}=\Omega_{1} r$, in the inner cylinder and $U_{\theta}=0$ in the outer cylinder. This is the most obvious choice. The component $U_{r}$ is set to zero at the boundaries. The velocity field is hereby continuous, but there exists a discontinuity of the velocity field derivative at the fluid boundaries (which is also the case in the real, physical situation). The error evolution with the penalization parameter and the convergence of the error with the resolution are shown in Figs. 5 and 6. For these calculations the expected $\sqrt{\eta}$ convergence order [22] is found and the convergence is second order in space as a function of the resolution $N$, confirming the results in [17]. We also observe a saturation of the convergence error for large $N$, corresponding to the penalization error.

A second way to impose the velocity in the mask will now be described. In this particular test-case the analytical solution is known (Eq. (26)) and we can use this information to increase the precision of the method. As mentioned in section 3.2, Gibbs oscillations are created due to discontinuities in the fluid variables or their derivatives. The discontinuity in the velocity gradient field can here be removed by using a $4^{\text {th }}$ order Hermite interpolation at the boundaries at $r=R_{1}, R_{2}$. Any purely azimuthal, axisymmetric flow is solenoidal so we can freely choose the velocity $U_{\theta}(r)$ in the mask, as long as it respects the correct boundary conditions at $r=R_{1}, r=R_{2}$ and $r=\pi$, the latter condition being imposed by the periodicity of the pseudo-spectral method. The velocity $U_{\theta}(r)$ in the mask is chosen such that velocity and derivative at the fluid-solid boundaries are continuous. Subsequently the velocity field is interpolated to 


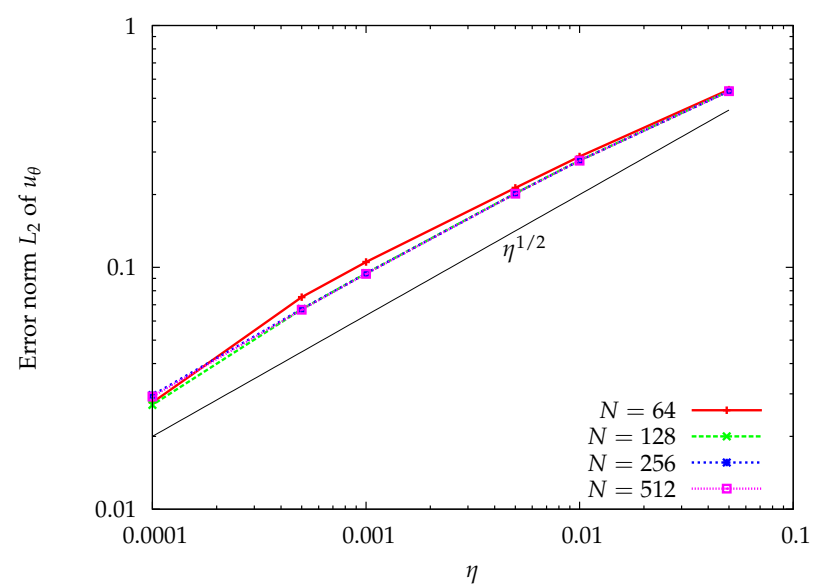

Figure 5: Convergence of the relative $L_{2}$ error of $u_{\theta}$ with the penalization parameter $\eta$. The convergence is shown to be of order $\sqrt{\eta}$.

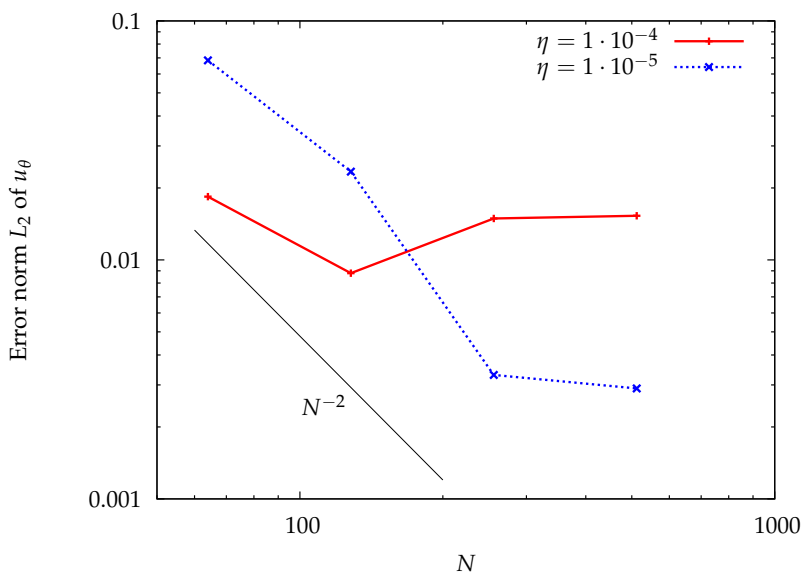

Figure 6: Convergence of the relative $L_{2}$ error of $u_{\theta}$ with the resolution $N$ which shows a second order convergence.

decay smoothly from the analytical solution at $r=R_{1}, R_{2}$ to zero at $r=0$ and $r=\pi$ respectively, using an interpolating Hermite polynomial. Any discontinuity on the derivative of the velocity field at the fluid boundaries is thus avoided and the Gibbs oscillations are hereby significantly reduced. In principle even higher order velocity derivatives could be smoothed in this way using higher order Hermite interpolation. Note that a similar regularization is used in [26] for the velocity field, where the imposed velocity in the mask is called an internal flow.

The error as a function of the penalization parameter and the convergence of the error with the resolution are shown respectively on Figs. 7 and 8. The numerical error is only calculated in the fluid domain. It is observed that the convergence of the error with the penalization parameter is close to third order. An optimum for the penalization parameter depending on the resolution appears, when the gridscale becomes of order $\sqrt{v \eta}$. At this scale the viscous term becomes of the order of the penalization term. In Figs. 7 and 8 results for both explicit and semi-implicit methods are used.

The regularization of the boundary conditions using an interpolation clearly improves the numerical convergence of the solution with the penalization parameter. Also the convergence with the grid resolution is improved. If the Hermite interpolation is used, a fourth order convergence with $N$ is found for both the explicit (dashed line) and semiimplicit (solid line) implementations (see Fig. 8). We recall that if no regularization of the velocity field is introduced, second order convergence is recovered (see Fig. 6).

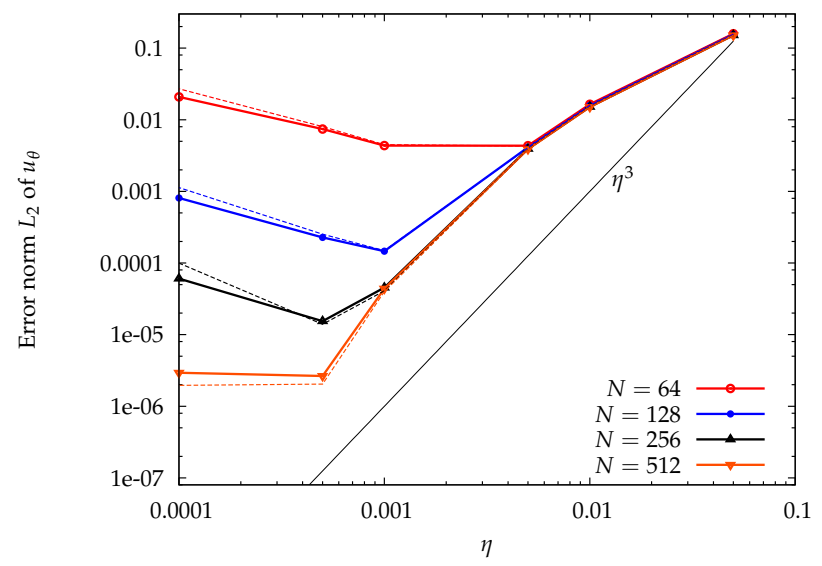

Figure 7: Convergence of the relative $L_{2}$ error with the penalization parameter $\eta$, semi-implicit (solid line) and explicit (dashed line) methods.

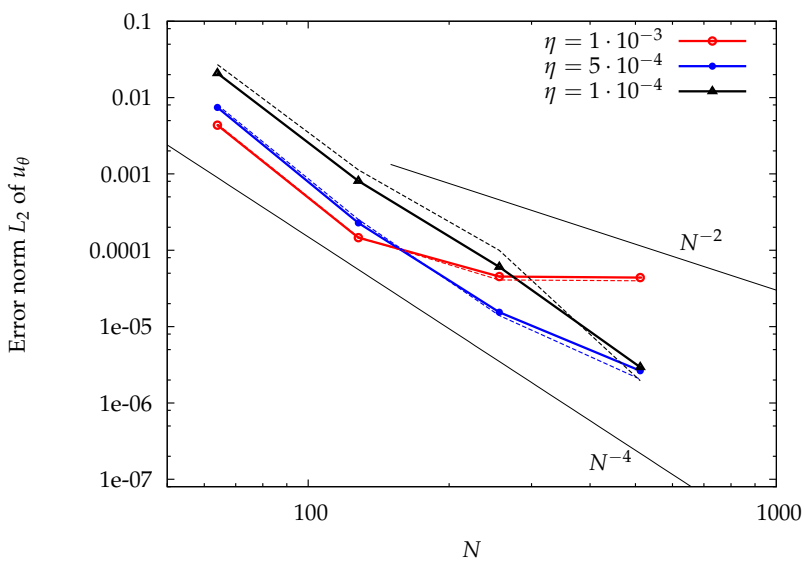

Figure 8: Convergence of the relative $L_{2}$ error with the resolution $N$, semi-implicit (solid line) and explicit (dashed line) methods. 


\subsection{The z-pinch}

The second validation test is the reproduction of the z-pinch phenomenon, a well-known textbook example of a confined plasma situation [27]. This configuration is illustrated in Fig. 9. Two ideal electrodes drive a 'toroidal' current in the $z$-direction producing a purely azimuthal magnetic field (in the $\theta$-direction). The current density in the $z$-direction, which together with the induced azimuthal magnetic field yield a radially pinching Lorentz force, is the motivation for the name $z$-pinch. In this configuration we set the velocity to zero so the code is entirely magnetic. We impose the boundary conditions $B_{\theta}=B_{C}$ and $B_{r}=0$ at $r=R_{1}$ the radius of the fluid domain. The component $B_{z}$ is not penalized and can freely evolve.

Periodic conditions are set in the axial direction. In this configuration the governing equations reduce to

$$
\begin{aligned}
\partial_{t} \boldsymbol{B} & =\lambda \nabla^{2} \boldsymbol{B}-\frac{\chi}{\eta}\left(\boldsymbol{B}-\boldsymbol{B}_{\text {wall }}\right) \\
\boldsymbol{J} & =\nabla \times \boldsymbol{B} \Rightarrow B_{\theta}=\frac{B_{C} r}{R_{1}}
\end{aligned}
$$

The computational domain is chosen similar to Fig. 2 (left). The mask is chosen to be annular, leaving the outer domain free to adapt to the periodic boundary conditions of the computational domain. If a uniform azimuthal magnetic field is imposed inside the mask, the discontinuity in the profile of the radial derivative of the azimuthal magnetic field at the boundary causes Gibbs' oscillations in the current density, analogously to what was observed in the TaylorCouette case. To avoid this, a linear profile of $B_{\theta}=\frac{B_{C} r}{R_{1}}$ is imposed inside the mask to ensure a continuity with the analytical solution. This feature eliminates the discontinuity at $r=R_{1}$ in the derivative of the magnetic field and thus greatly reduces the oscillations for $J_{z}$. The convergence of the method can be further enhanced by using a Hermite polynomial to smoothly interpolate the magnetic field in the mask to zero at the outer boundaries of the computational domain. With this method, the continuity of the derivative of the magnetic field is assured through the entire domain (see Fig. 2 (right)).

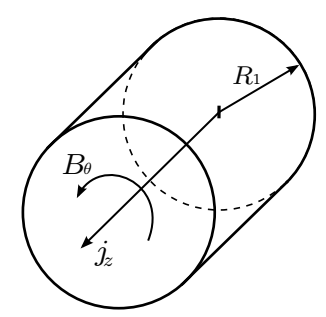

Figure 9: $z$-pinch configuration.

For these simulations, the parameters are a cubic domain with size-length $2 \pi$ with $\lambda=1$, the time step is fixed to $\Delta t=5.10^{-5}$ and $t_{\max }=5$. For $t=t_{\max }$ the difference in the magnetic energy between two iterations is less than $10^{-9}$ so we have reached the steady state. The inner radius of the annulus is $R_{1}=0.65 \pi$ and the outer radius is $R_{2}=0.78 \pi$. If the Hermite polynomial is used, the radius where it reaches the value 0 is $R_{3}=0.94 \pi$. The boundary condition $B_{C}=1$.

In Fig. 10 the convergence of the method is shown as a function of the penalization parameter $\eta$ and the resolution $N$. An improved convergence, proportional to $\eta^{4}$ or $N^{-4}$ is observed. The relative importance of the smoothing of the magnetic field in the mask is illustrated in Fig. 10 where the results of these computations with and without regularization are compared. Without the regularization technique, the convergence reduces to second order in $N$ and order $\sqrt{\eta}$ for the penalization parameter.

\subsection{Assessment of the regularization method to enhance the performance of the penalization method}

For these two-dimensional test cases, either Taylor-Couette or $z$-pinch, the error of convergence as a function of the penalization parameter and the resolution are determined. The regularization of the different fields in the solid domain (or mask) allows an enhancement of the accuracy of the numerical solution in the fluid region. The fact that the continuity of the velocity derivative field inside the solid domain affects the error inside the fluid domain is an 

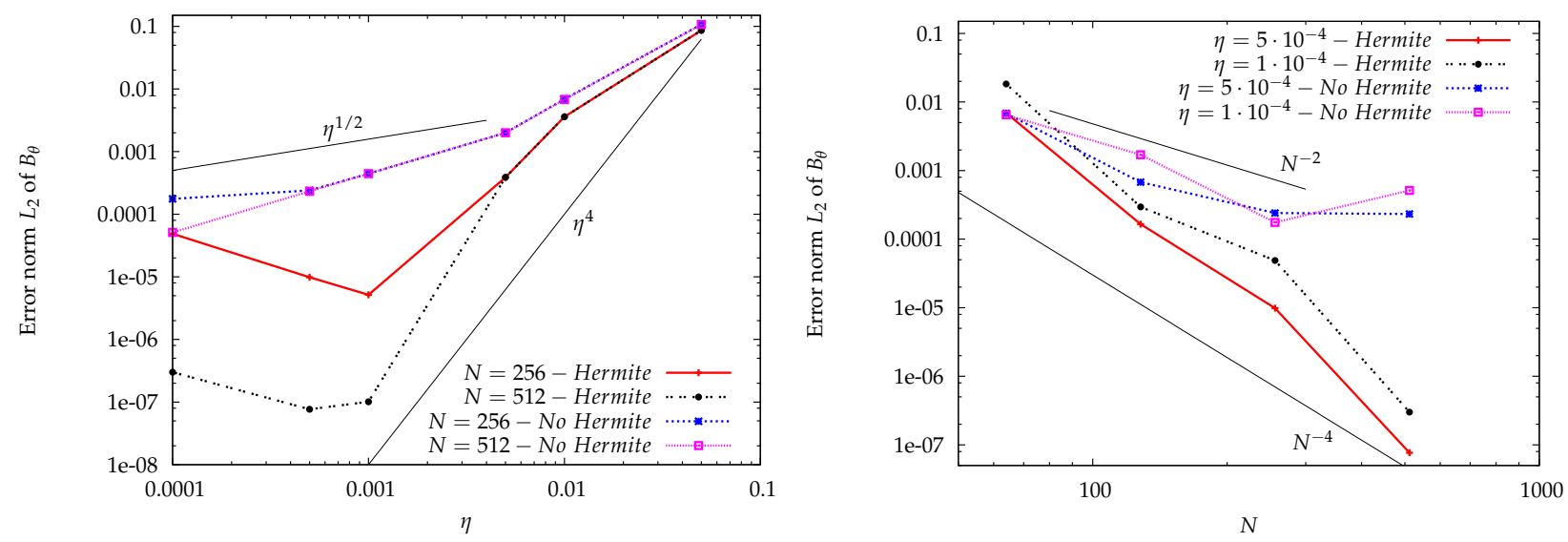

Figure 10: Convergence of the relative $L_{2}$ error with the penalization parameter $\eta$ (left) and with the resolution $N$ (right) for the magnetic field in the $z$-pinch geometry. Comparison of the results with and without Hermite polynomial interpolation.

intrinsic feature of the pseudo-spectral method. Such methods use periodic trial functions and the Gibbs oscillations introduced in one point of the domain decay only inversely proportional to the distance from the discontinuity. The Hermite interpolation method regularizes and yields fields which are $C^{1}$ in the whole domain. Gibbs oscillations are thus reduced and consequently the numerical errors are also decreased considerably. The limitation of this method is that an analytical solution must be known, or a baseflow, which is not far from the expected developed flow. Without such regularization the convergence is reduced to second order in resolution, which can be sufficient for many applications, as illustrated in the following sections.

\section{Three-dimensional validation}

In this section the code will be validated by considering three-dimensional test-cases. First a periodic MHD case is considered, without using the penalization method, subsequently the magnetic part of the code is validated by studying the ohmic decay in a cylindrical cavity. Then the three-dimensional Taylor-Couette flow is studied with and without the presence of a magnetic field and to conclude we investigate the instabilities in a cylinder with helical magnetic boundary conditions.

\subsection{Periodic MHD validation}

To validate the capacity of the numerical code to simulate the three-dimensional nonlinear MHD equations, we reproduce first a classical test-case with periodic boundary conditions. This case is the generalization of the OrszagTang vortex to three dimensions. The results are compared with those of Mininni et al. [28].

The initial condition used for the simulation is given analytically and yields:

$$
\boldsymbol{u}(x, y, z, t=0)=[-2 \sin (y), 2 \sin (x), 0] \text { for } x, y, z \in[0,2 \pi]^{3}
$$

and

$$
\boldsymbol{B}(x, y, z, t=0)=\beta[-2 \sin (2 y)+\sin (z), 2 \sin (x)+\sin (z), \sin (x)+\sin (y)]
$$

with $\beta=0.8$, the initial kinetic energy, $E_{k}=2$, and the corresponding magnetic energy, $E_{m}=1.92$. The energies are evaluated by $E_{k}=\frac{1}{2}\left\langle\mathbf{u}^{2}\right\rangle$ and $E_{m}=\frac{1}{2}\left\langle\mathbf{B}^{2}\right\rangle$ with $\langle.$.$\rangle the volume average. The maximum of the current density is$ calculated by

$$
\max |j|=\max \sqrt{j_{x}^{2}+j_{y}^{2}+j_{z}^{2}}
$$

and the total dissipation rate is

$$
\varepsilon(t)=v\left\langle\omega^{2}\right\rangle+\lambda\left\langle j^{2}\right\rangle
$$



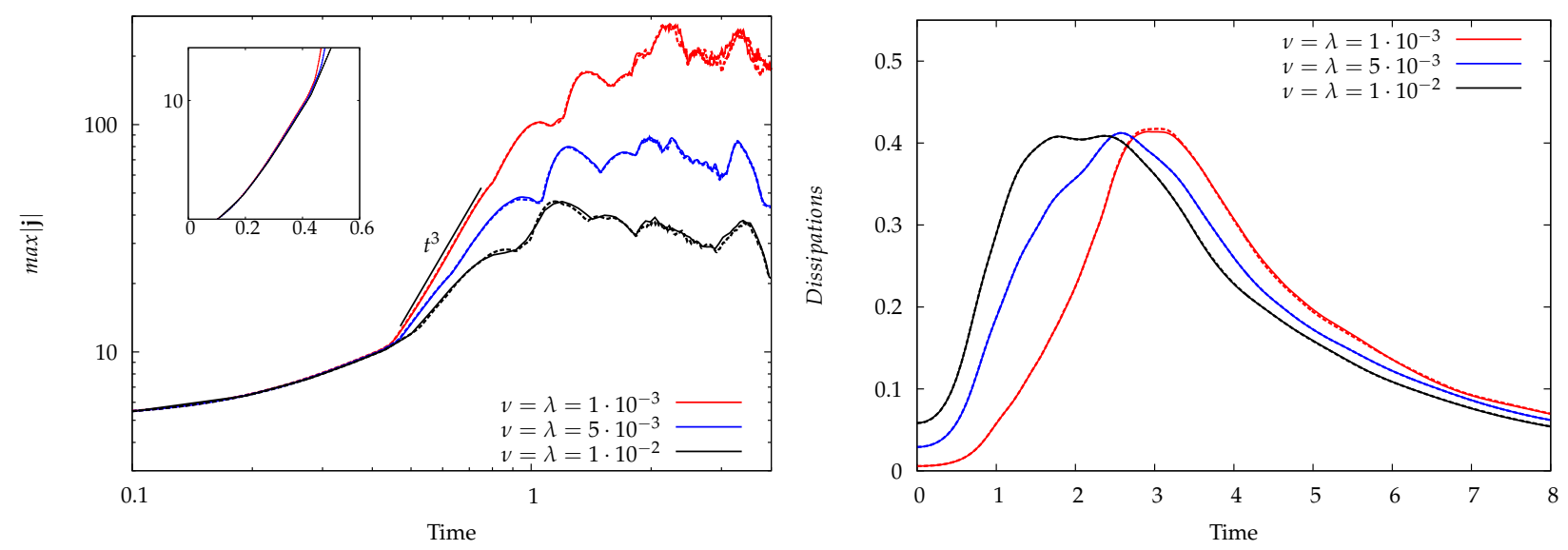

Figure 11: Comparison of the solution of the numerical code for the time evolution of the maximum of the current (left) and total dissipation rate (right) using second (dashed line) and third order time-schemes (solid line). The inset (left) shows the evolution at early times in lin-log units.

where $v$ and $\lambda$ are respectively the kinematic viscosity and the magnetic diffusivity. Three runs are performed: the first with $N^{3}=64^{3}$ and $v=\lambda=0.01$, the second with $N^{3}=128^{3}$ and $v=\lambda=0.005$ and the third $N^{3}=256^{3}$ with $v=\lambda=0.001$.

The evolution of the maximum of the current density and total dissipation rate in the domain are shown in Fig. 11. Results are compared using a second- and third-order Adams-Bashforth time advancing scheme described in section 3.3. Both schemes give the same results. The results agree well with the computations presented in reference [28]. The same exponential growth followed by a self similar growth $\sim t^{3}$ is found in our calculations for the evolution of the maximum current density (see Fig. 11 (left)). With an increasing Reynolds number Re the maximum of $|j|$ is also found to be reached at later times. The evolution of the total dissipation rate (see Fig. 11 (right)) shows the same delay in the onset of the formation of small scales with increasing $R e$ as exposed in the cited article. This test allows us to evaluate the full MHD code and validate the numerical results for relatively high Reynolds numbers (up to $R e=3000)$.

\subsection{Ohmic decay in a periodic cylinder}

In this test case we compute the evolution of the magnetic field in a periodic three dimensional cylinder coated with an insulator $[29,30]$. In the induction equation we set the velocity to zero so the equation for the magnetic field reduces to the diffusion equation:

$$
\frac{\partial \boldsymbol{B}}{\partial t}=\nabla^{2} \boldsymbol{B}
$$

We consider an axisymmetric case, $z$-independent and the magnetic field has no $r$ component. This case is not completely three-dimensional since we use the three components of the magnetic field $\left(B_{x}, B_{y}, B_{z}\right)$, but the derivatives are zero in the $z$ direction. In cylindrical coordinates the set of equations is:

$$
\left\{\begin{array}{l}
\frac{\partial B_{\theta}}{\partial t}=\frac{\partial^{2} B_{\theta}}{\partial r^{2}}+\frac{1}{r} \frac{\partial B_{\theta}}{\partial r}-\frac{B_{\theta}}{r^{2}} \\
\frac{\partial B_{z}}{\partial t}=\frac{\partial^{2} B_{z}}{\partial r^{2}}+\frac{1}{r} \frac{\partial B_{z}}{\partial r} .
\end{array}\right.
$$

Using separable elementary solutions, the magnetic field can be written in the following form:

$$
\left\{\begin{array}{l}
B_{\theta}(r, t)=A f(r) e^{-\omega_{\theta}^{2} t} \\
B_{z}(r, t)=B g(r) e^{-\omega_{z}^{2} t}
\end{array}\right.
$$

Introducing the following change of parameter: $s=\omega r$ the system of equations writes:

$$
\left\{\begin{array}{l}
s_{\theta}^{2} f^{\prime \prime}+s_{\theta} f^{\prime}+\left(s_{\theta}^{2}-1\right) f=0 \\
s_{z}^{2} g^{\prime \prime}+s_{z} g^{\prime}+s_{z}^{2} g=0
\end{array}\right.
$$


The solutions of these equations are Bessel functions. Imposing at the radius $R_{0}$ of the cylinder the azimuthal and axial field vanishing the general solution is:

$$
\left\{\begin{array}{l}
B_{\theta}(r, t)=J_{1}\left(\frac{j_{1}}{R_{0}} r\right) e^{-\left(\frac{j_{1}}{R_{0}}\right)^{2} t} \\
B_{z}(r, t)=J_{0}\left(\frac{j_{0}}{R_{0}} r\right) e^{-\left(\frac{j_{0}}{R_{0}}\right)^{2} t} .
\end{array}\right.
$$

Here $j_{0}=2.4048 \ldots$ and $j_{1}=3.8314 \ldots$ are the first zeros of the Bessel functions $J_{0}$ and $J_{1}$ respectively.

In our simulation we set $R_{0}=1$. The decay rate is determined doing a least square fitting of the azimuthal and axial magnetic energy time evolutions. In table 2 we present our results for the decay rate of the azimuthal and axial fields and we compare them to the analytical values (see Eq. (37)). All the simulations are done with $96^{3}$ grid points and the penalization parameter $\eta=5 \cdot 10^{-4}$. We calculate the error for different fixed time steps. In none of these calculations the regularization of the magnetic field in the solid region (mask) is used.

\begin{tabular}{l|ccc|ccc}
\hline Time step & $\begin{array}{c}\text { Theory } \\
\omega_{\theta}^{2}\end{array}$ & $\begin{array}{c}\text { Numerical } \\
\omega_{\theta}{ }^{2}\end{array}$ & $\begin{array}{c}\text { Error } \\
\omega_{\theta}{ }^{2}\end{array}$ & $\begin{array}{c}\text { Theory } \\
\omega_{z}{ }^{2}\end{array}$ & $\begin{array}{c}\text { Numerical } \\
\omega_{z}^{2}\end{array}$ & $\begin{array}{c}\text { Error } \\
\omega_{z}^{2}\end{array}$ \\
\hline $1 \cdot 10^{-2}$ & & 12.68 & $14 \%$ & & 4.99 & $14 \%$ \\
$1 \cdot 10^{-3}$ & \multirow{3}{*}{14.68} & 13.99 & $5 \%$ & 5.78 & 5.51 & $5 \%$ \\
$5 \cdot 10^{-4}$ & 14.13 & $4 \%$ & & 5.57 & $4 \%$ \\
$1 \cdot 10^{-4}$ & & 14.28 & $3 \%$ & & 5.62 & $3 \%$ \\
\hline
\end{tabular}

Table 2: Comparison of analytical (see Eq. (37)) and numerical calculation of the decay rate for the ohmic diffusion in an infinite cylinder.

For these calculations of the diffusion of a magnetic field in a periodic cylinder the decay rate of the azimuthal and axial component of the magnetic field agree quite well with the analytical values. We find the same relative error in the azimuthal and in the axial direction at each considered time discretization.

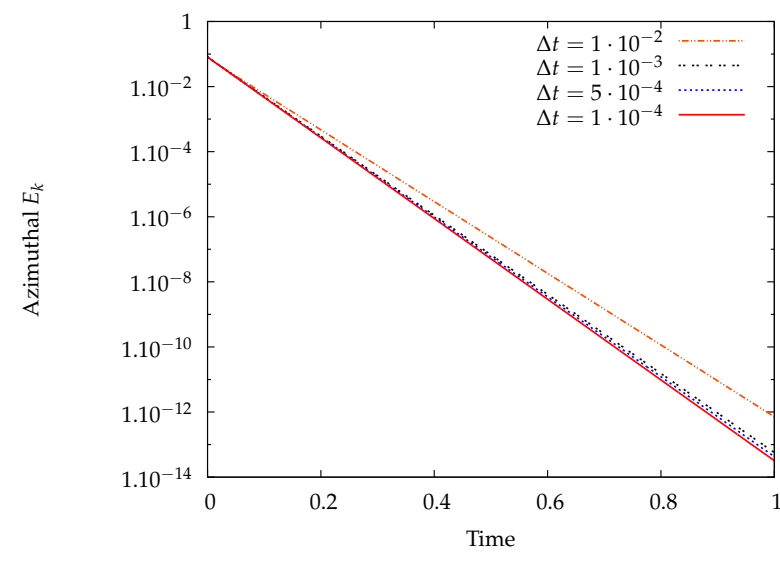

Figure 12: Azimuthal magnetic energy decay for different time steps in an periodic cylinder.

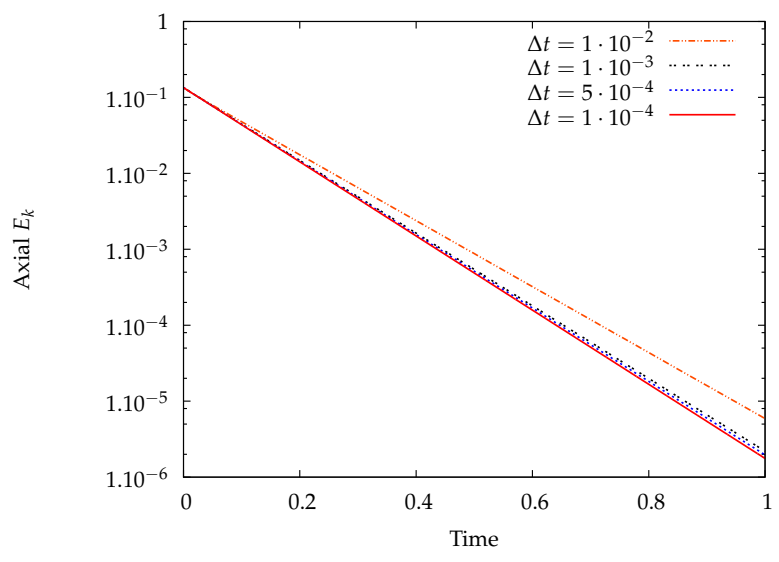

Figure 13: Axial magnetic energy decay for different time steps in an periodic cylinder.

This test-case yields a validation of the magnetic part of the numerical code and shows that the magnetic boundary conditions are well taken into account via the penalization method. Also we note that the time scheme is well implemented as it allows to recover the analytical decay rates for the considered components of the magnetic field with good accuracy. The numerical solution converges towards the analytical solution if the time step is decreased.

\subsection{Three-dimensional Taylor-Couette flow}

In this test case we aim to determine the critical Reynolds number for the first instability of the Taylor-Couette flow with periodic boundaries in the axial direction. Different values of the Reynolds number are explored with 
several calculations with a resolution of $128^{3}$ grid-points and the penalization parameter $\eta=5 \cdot 10^{-4}$. In none of these calculations the regularization of the velocity in the solid region (mask) is used. The reference length scale is the gap between the inner and outer cylinder $\mathcal{L}=R_{\text {ext }}-R_{\text {int }}$ and the reference velocity is the inner rotation speed $U=\Omega_{\text {int }} R_{\text {int }}$. The outer cylinder is fixed. We define the Reynolds number and also a radius ratio $\zeta$ and a aspect ratio $\Gamma$ as follows:

$$
R e=\frac{U \mathcal{L}}{v}, \quad \zeta=\frac{R_{\text {int }}}{R_{\text {ext }}}, \quad \quad \Gamma=\frac{L_{z}}{\mathcal{L}},
$$

where $L_{z}$ is the axial length. To be able to compare with the literature we take the same values as in [31] for the dimensionless values, radius ratio and aspect ratio, $\zeta=0.5$ and $\Gamma=4$. The base flow consists of an azimuthal velocity only, as in the two-dimensional case (section 4.1). The first Taylor-Couette instability is centrifugal and is characterized by vortices that appear and break the axial invariance. Velocities in the radial and axial directions thereby appear. To determine the critical Reynolds number we analyze the evolution of the axial kinetic energy. The analysis of the evolution of the axial kinetic energy allows us to assess the critical Reynolds number (when the instability is triggered). The value of the critical Reynolds is compared with a theoretical value of $R e=68.23$ that has been determined by Chandrasekhar [32]. We present in Fig. 14 the axial kinetic energy evolution for Reynolds numbers varying from $R e=65.7$ to $R e=69.7$. We start the simulations with a small random perturbation so the initial axial kinetic energy is non zero. The axial energy either grows or decays exponentially. The critical Reynolds number can be determined from Fig. 14. Increasing the Reynolds number from $R e=67.3$ and $R e=68.1$ the axial kinetic energy changes from decaying to increasing. A simple linear interpolation of the growth and decay rates (that are determined with a least square method fitting) gives the value of the critical Reynolds $R e_{c} \approx 67.9$. The estimated error compared with the theoretical result is $\sim 0.44 \%$. The theoretical estimate is thus well approached by our numerical simulations. The resolution can be increased further which should allow, in principle, to approach even more closely the instability threshold.

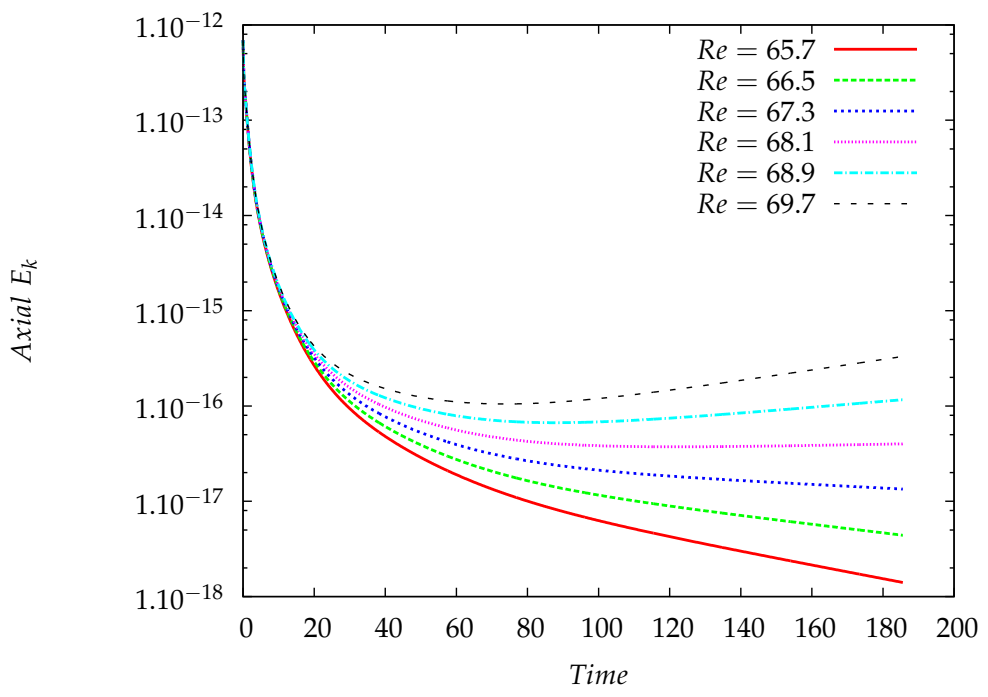

Figure 14: Evolution of the axial kinetic energy for different $R e$ numbers.

The Taylor vortices appear early in the simulation and they grow or decay in strength depending on the Reynolds number. Fig. 15 presents the axial kinetic energy evolution of a Taylor-Couette flow for $R e=120$. When the nonlinear saturation is reached a steady state is obtained. In Figs. 16 to 19 we visualize the Taylor vortices in the steady state. Two pairs of counter-rotating vortices appear. The aspect ratio is $\Gamma=4$ so that four vortices form. In Fig. 17 we distinguish the boundary layer, in which the azimuthal vorticity is contrary to the vorticity of the Taylor-vortices. Also some small Gibbs oscillations are present and visible at the border of the fluid domain. The above results have been obtained with the L-code. Those of the M-code are almost identical and are therefore not shown.

The flow structure of Figs. 16 to 19 can be compared with the one presented by Guermond et al. (Fig. 5 [31]). The same flow topology with four vortices is found. In Figs. 18 and 19 we note that there is a transport of azimuthal 


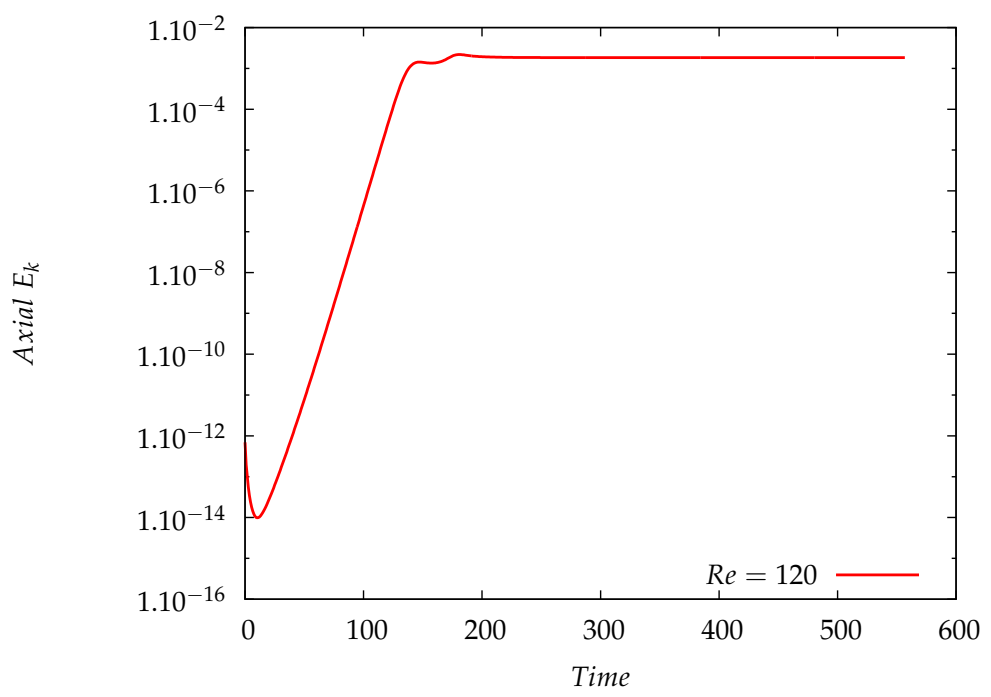

Figure 15: Evolution of the axial kinetic energy for $R e=120$.

momentum by the radial flow. The azimuthal velocity isosurfaces are not axial invariant but they are dragged in- and outwards by the radial flow. A positive radial velocity increases the azimuthal velocity near the inner cylinder.

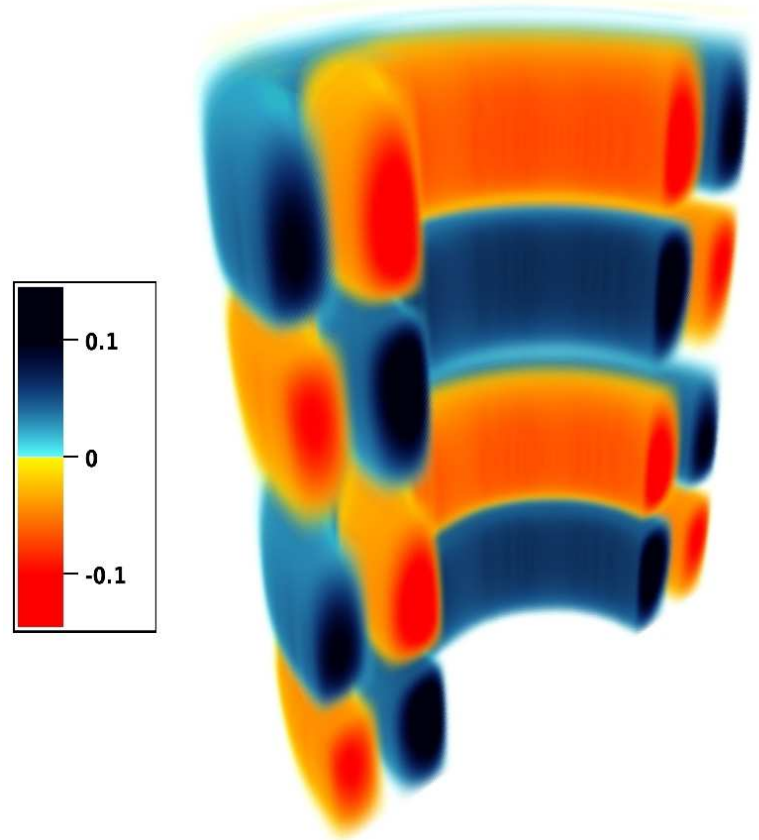

Figure 16: Axial velocity $u_{z}$ for $R e=120$.

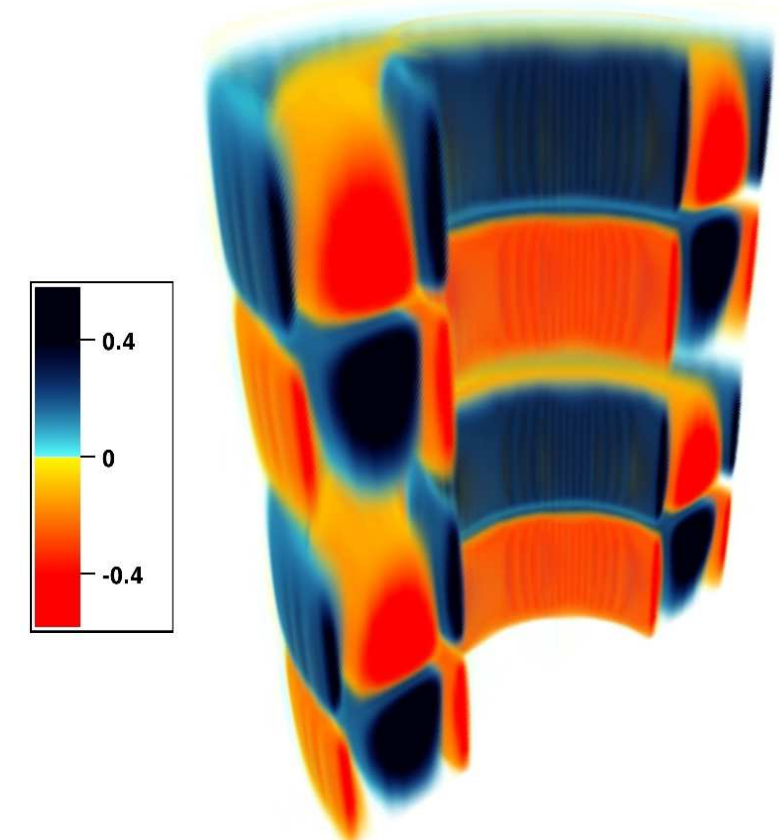

Figure 17: Azimuthal vorticity $\omega_{\theta}$ for $R e=120$.

To compare quantitatively both simulations, in table 3 the maxima of the three velocity components at the steady state are compared with those of Guermond et al. [31].

The azimuthal velocity is not exactly unity because with the penalization method the precise value at the boundary is not necessarily coinciding with the numerical grid as is the case in [31]. Nevertheless the numerical method yields good agreement for all three components of the velocity with the results of the code described in [31]. 


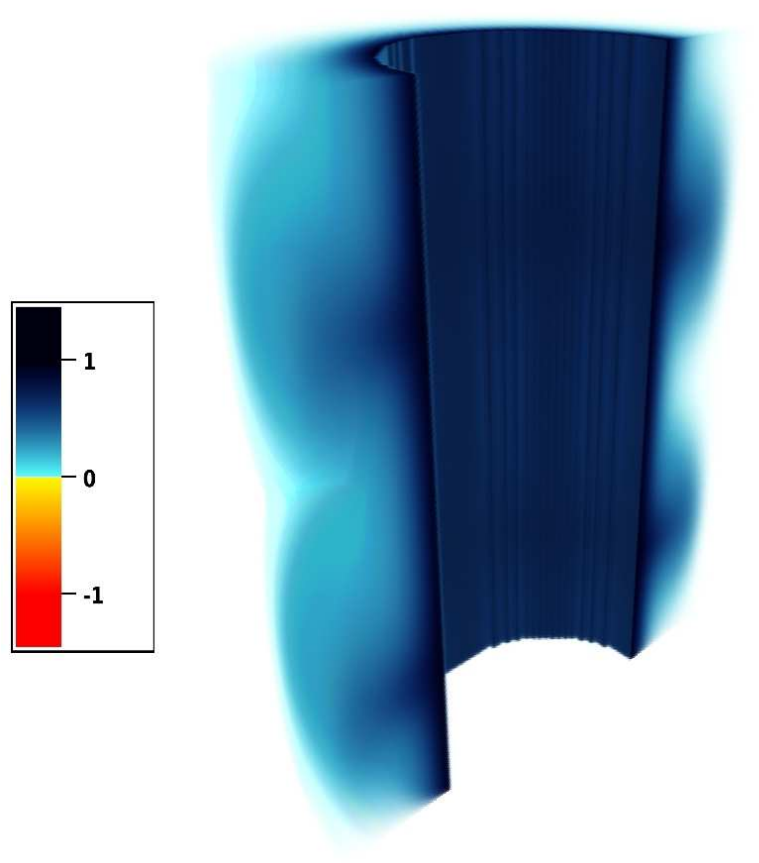

Figure 18: Azimuthal velocity $u_{\theta}$ for $R e=120$.

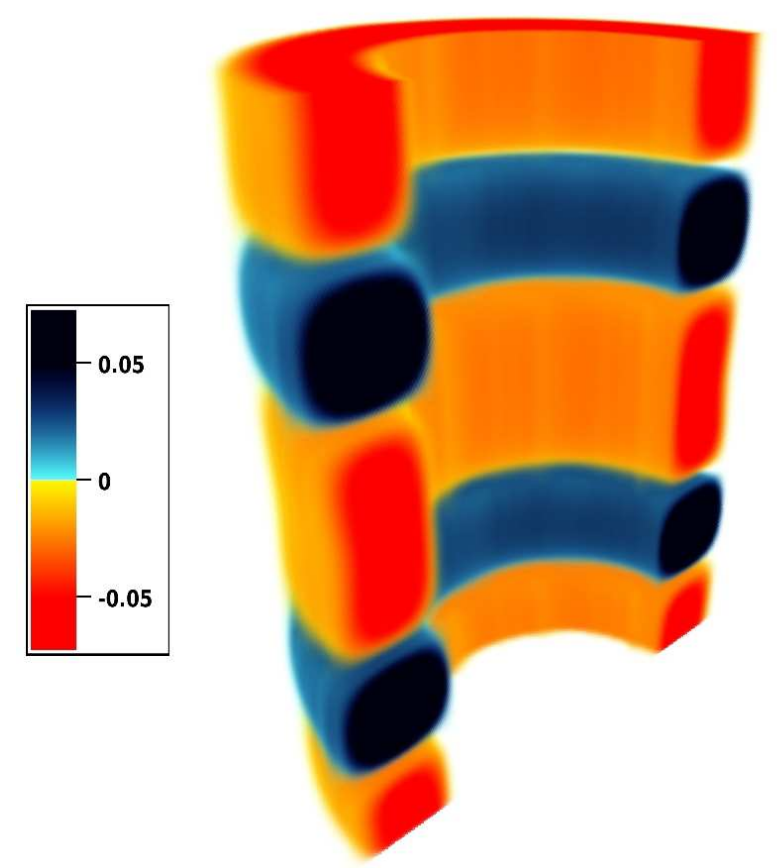

Figure 19: Radial velocity $u_{r}$ for $R e=120$.

\begin{tabular}{llll}
\hline & Guermond et al. [31] & 3rd order semi-implicit & 2nd order explicit \\
\hline $\max u_{r}$ & 0.1935 & $0.19434(\sim 0.43 \%)$ & $0.1969(\sim 1.75 \%)$ \\
\hline $\max u_{\theta}$ & 1 & $0.99693(\sim 0.31 \%)$ & $0.9980(\sim 0.20 \%)$ \\
\hline $\max u_{z}$ & 0.1454 & $0.14639(\sim 0.68 \%)$ & $0.1506(\sim 3.57 \%)$ \\
\hline
\end{tabular}

Table 3: Maximum velocity components comparison and relative errors for $R e=120$ in a periodic cylinder.

With this test-case the well known linear first instability threshold of the Taylor-Couette flow is found. Also the nonlinear saturation is comparable to what is reported in the literature. The same topology of the flow is observed and quantitatively similar velocity magnitudes at the steady state are established. This makes us confident about the accuracy of the method in taking into account centrifugal, pressure and nonlinear effects in a bounded domain.

\subsection{Magnetohydrodynamic Taylor-Couette flow}

We now extend our validation to the test-case of the instability of an axisymmetric MHD Taylor-Couette flow with periodic boundary conditions in the axial direction. In this case the instability studied in the previous section is modified due to the presence of an axial magnetic field. An imposed constant axial field $B_{0}$ is added to the magnetic field. It is well known that such an axial magnetic field has a significant stabilizing effect. This phenomenon of delay in the appearance of the first Taylor-Couette instability was found by Chandrasekhar [32], confirmed by linear numerical calculations in [7] and by spectral numerical simulations [9, 33]. The fluid flow will try to bend the axial magnetic field lines but the restoring Lorentz force will prevent the fluid motion, which stabilizes the flow profile.

To assess our numerical codes in this context, we evaluate the evolution of the axial kinetic energy as a function of the Hartmann number $\mathrm{Ha}$, which measures the ratio between electrodynamic forces and viscous forces. The presence of a uniform magnetic field in the axial direction does not affect the profile of the stable azimuthal velocity that exists without the magnetic field, Eq. (26), which we choose as initial condition. The dimensionless geometric parameters, radius ratio $\zeta$ and aspect ratio $\Gamma$ are the same as in the previous section. We introduce here the magnetic Prandtl number which is the ratio of viscosity and magnetic diffusivity. The dimensionless numbers describing the problem 
are then

$$
\operatorname{Pr}=\frac{v}{\lambda}, \quad \operatorname{Re}=\frac{U \mathcal{L}}{v}, \quad H a=\frac{B_{0} \mathcal{L}}{\sqrt{\mu_{0} \rho v \lambda}}, \quad \zeta=\frac{R_{\text {int }}}{R_{\text {ext }}}, \quad \Gamma=\frac{L_{z}}{\mathcal{L}},
$$

where $\mu_{0}$ is the magnetic constant and $\rho$ is the fluid density. The simulations are performed for $\operatorname{Pr}=1, \operatorname{Re}=100$, $\zeta=0.5$ and $\Gamma=4$. The resolution used is $N^{3}=128^{3}$ and the penalization parameter $\eta=5 \cdot 10^{-4}$. For none of these calculations regularization of the velocity or magnetic field in the solid region (or mask) is used. The boundary conditions described in [7] are a fixed azimuthal velocity on the inner cylinder $\left(U=\Omega_{\text {int }} R_{\text {int }}=1\right)$, no-slip on the outer cylinder and perfectly conducting walls, so that the normal magnetic field at the wall and the axial current density vanish $\left(b_{r}=0\right.$ and $\left.j_{z}=0\right)$ [7]. With these parameters the Taylor-Couette flow is hydrodynamically unstable, as was observed in the previous section. With the penalization method we can impose the vanishing radial magnetic field but the current density is not constrained.

The evolution of the axial kinetic energy varies as a function of the imposed magnetic field (or Hartmann number), for a fixed Reynolds number, as is shown in Fig. 20. These calculations allow us to determine the threshold for the instability. For $R e=100$ the critical Hartmann number we find is $H a_{c} \approx 7.9$. Like in the previous section the threshold is determined by linear interpolation of the growth and decay rates. For $R e=100$ the flow is hydrodynamically unstable and Taylor vortices should appear, but for $\mathrm{Ha}>7.9$, the instability is suppressed by the magnetic field.

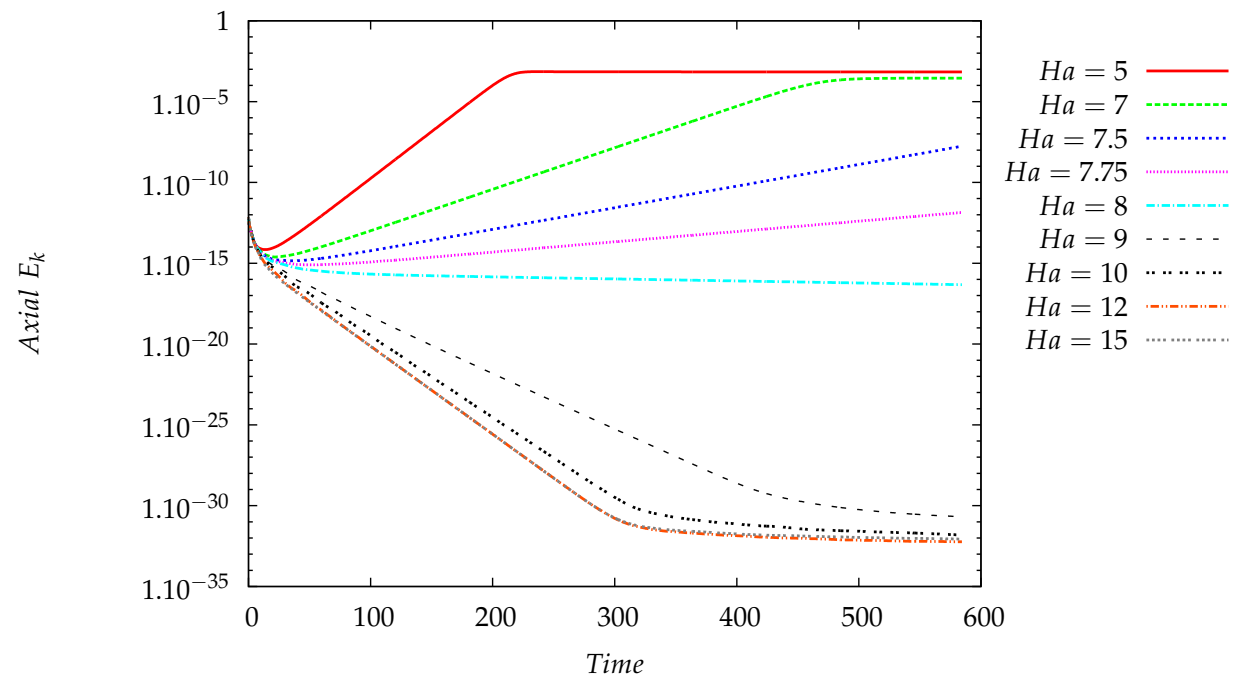

Figure 20: Evolution of the axial kinetic energy for different Hartmann numbers.

In Fig. 21 we show the topology of the flow resulting of our simulation (cut in the $r-z$ plane). This figure could be compared to Fig. 1 in [9]. This comparison is merely qualitative, since the ratio of the radii is $\zeta=0.95$ in the cited reference and here we have used $\zeta=0.5$. We have not tried to quantitatively study the same geometry, since our method is not particularly adapted for the case $\zeta=0.95$, because a very large part of the computational domain would correspond to the mask. To obtain a reasonable number of grid-points in the fluid domain, extremely large resolutions would be needed. Immersed boundary methods with uniform space discretization are clearly not the most adapted tool for this aspect ratio. The parameters chosen in our simulation are $\operatorname{Pr}=1, \mathrm{Ha}=7, \operatorname{Re}=100, \zeta=0.5$ and $\Gamma=4$. Despite the different parameters for the two computations, the resulting hydromagnetic flow has a similar topology. We note how the magnetic lines are advected by the flow. The resulting restoring Lorentz force stabilizes the fluid.

With this test-case we therefore found the well known stabilizing effect of an axial magnetic field on the TaylorCouette flow. Qualitatively the flow behavior is very similar to what is found in other investigations. We were not able to quantitatively compare with the literature since our boundary conditions on the magnetic field are not the same as those considered in previous studies on magnetohydrodynamic Taylor-Couette flow and the parameter $\zeta$ was different. To compare with more accuracy our codes with the literature we treat in the following section a test case with boundary conditions and geometry which are adapted to our numerical method. 

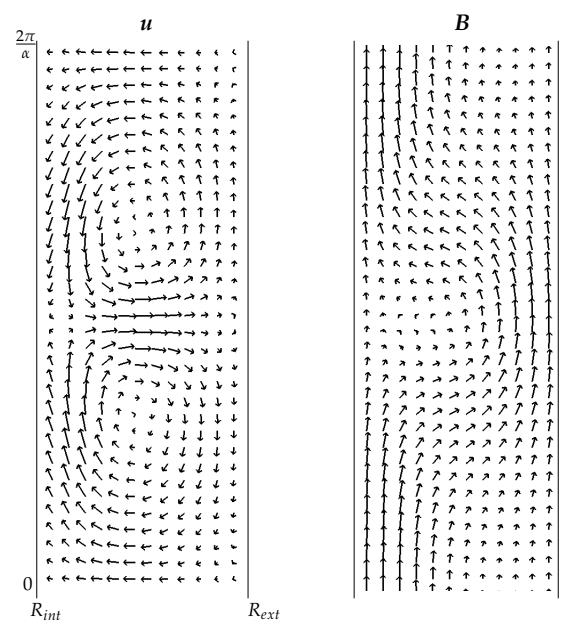

Figure 21: Hydromagnetic flow. Cut in the $r-z$ plane of velocity $\boldsymbol{u}$ (left) and magnetic (B) field (right) for $\operatorname{Pr}=1, \mathrm{Ha}=7, \operatorname{Re}=100, \zeta=0.5$ and $\Gamma=4$.

\subsection{Flow induced by a helical magnetic field}

Shan, Montgomery and Chen [34] studied numerically a conductive fluid confined in a periodic cylinder where an axial electric and magnetic field are imposed, which results in a helical magnetic field (see Fig. 22). They used a spectral code which decomposed the fields onto Chandrasekhar-Kendall orthonormal eigenfunctions of the curl. In that study they discovered a transition between an axisymmetric state with a zero velocity to a laminar helical state where a dynamic equilibrium appears, i.e., a steady state with non zero velocity.

The parameters chosen for the numerical study are selected to closely reproduce the simulations in [34]. A fixed axial magnetic field $B_{0}=4.5$ is imposed and the fluid has a constant magnetic diffusivity and kinematic viscosity $\lambda=v=0.045$. The computational domain size is $L_{x}=L_{y}=0.8 \pi$ and $L_{z}=8$. The typical length scale is the cylinder radius, $\mathcal{L}=R_{0}=1$, the axial length is $L_{z}=8 R_{0}$, the resolution used for the simulations is $N^{3}=128^{3}$ grid-points and the penalization parameter $\eta=5 \cdot 10^{-4}$. Three dimensionless numbers characterize the system: the Lundquist number $(\mathrm{S})$, the Hartmann number $(\mathrm{Ha})$ and the pinch ratio for the axisymmetric zero flow state $\left(\Theta_{0}\right)$ :

$$
S=\frac{C_{A} \mathcal{L}}{\lambda}, \quad H a=\frac{B_{0} \mathcal{L}}{\sqrt{\rho \mu_{0} \lambda \nu}}, \quad \Theta_{0}=\frac{\overline{B_{\theta}}}{\left\langle B_{z}\right\rangle},
$$

with $C_{A}$ the axial Alfvén velocity $C_{A}=B_{0} / \sqrt{\rho \mu_{0}}, \overline{B_{\theta}}$ is the wall-averaged poloidal magnetic field and $\left\langle B_{z}\right\rangle$ is the volume-averaged axial magnetic field.

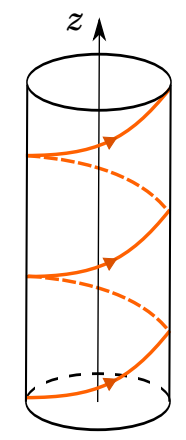

Figure 22: Helical magnetic field scheme.

The transition between states is determined as a function of one of these dimensionless numbers, the pinch ratio $\Theta_{0}$, which is varied by adjusting the imposed average axial electric field $E_{0}$ and which is directly linked to the poloidal 
magnetic field. The other dimensionless numbers are maintained constant, $S=H a=100$. For the parameters given above, the linear theory predicts a transition for an imposed electric field $E_{0}=0.33[34,35]$.

To compare with Shan et al. we impose the same boundary conditions. The walls are treated as perfect conductors and are coated with a thin layer of insulator. Hereby both the radial magnetic and current density field vanish $\left(B_{r}=\right.$ $j_{r}=0$ ). For the velocity field only the radial component vanishes at the wall. The penalization method is used to impose a vanishing normal component of the velocity $(\boldsymbol{u} \cdot \boldsymbol{n}=0)$ without any regularization technique. Also an azimuthal magnetic field $B_{\theta_{0}}$ is imposed via the penalization term. In this case the vanishing radial current density $\left(j_{r}=0\right)$ is automatically satisfied because the azimuthal magnetic field does not generate a radial current density (the $r$-component of the curl of the imposed magnetic field is zero). The boundary conditions are thus satisfied.

The way the electric field is imposed in our simulations differs from the simulations by Shan et al. In their simulations the electric field explicitly appears in the discretized equations, whereas in our case the electric field is indirectly imposed through the magnetic field at the wall (which can be related to the electric field using Stokes' theorem). This can lead to small differences in the transients, but is not expected to greatly affect the steady state solutions.

The azimuthal magnetic field $B_{\theta_{0}}(r)$ is imposed with the volume penalization method in the solid region using the regularization technique, like for the $z$-pinch case (see section 4.2). The azimuthal magnetic field increases linearly with $r$ from $r=R_{0}$ (the fluid-solid frontier) to $r=0.34 \pi$ and then smoothly tends to zero using a Hermite's interpolating polynomial from $r=0.34 \pi$ to $r=0.385 \pi$. The periodicity of the computational region is hereby satisfied and the magnetic derivative of the base-field is continuous at the wall.

To validate the code we perform the same calculations done by Shan et al.. We vary the electric field $E_{m}$, hence the axial current density $\left(j_{z}\right)$, and we calculate $E_{0}$ when the simulation reaches a steady state using Ohm's law

$$
E_{0}=(-\boldsymbol{u} \times \boldsymbol{B})_{z}+\frac{j_{z}}{\sigma}
$$

We find (see Fig. 24) that the instability threshold between the axisymmetric and helical state is situated between $E_{0}=0.302$ and $E_{0}=0.355$ as found in [34]. The kinetic energy starts to grow when the imposed electric current is $E_{0}=0.355$. The growth rate of the energy is calculated using Alfvén time units $\left(t_{A}=t_{\text {num }} C_{A} / L_{z}\right)$. A least-square fitting gives the growth rate of the kinetic energy as 0.54 , corresponding to a growth rate of 0.27 . This can be compared to the analytical value 0.279 calculated for an applied electric field $E_{0}=0.35$. Taking into account that our imposed magnetic field is slightly different (since the electric field is imposed indirectly in our case), the two different growth rates match in good order. In Fig. 23 the excited mode is visualized, which is a helical mode with azimuthal and axial mode numbers $m=n=1$, respectively, as in [34].

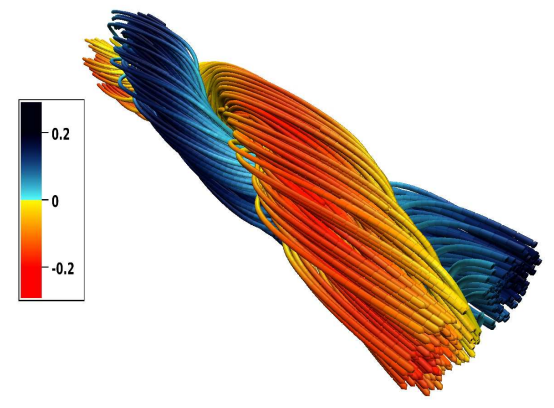

Figure 23: First helical mode, velocity streamlines colored with the axial velocity $u_{z}$.

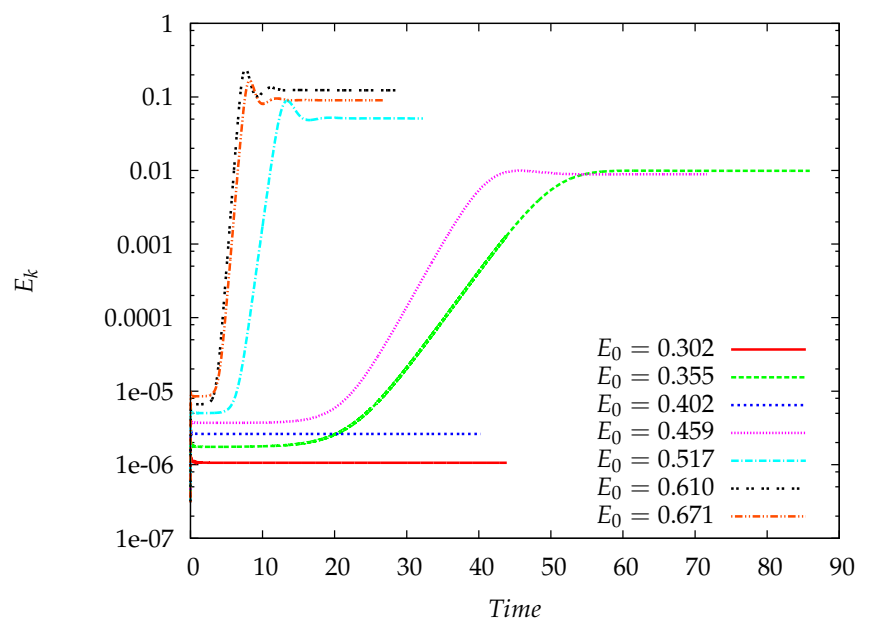

Figure 24: Evolution of the kinetic energy for different imposed axial electric fields. 
Increasing the pinch ratio to $E_{0}=0.402$ the flow returns to its axisymmetric copper-wire solution, which is also observed for certain values in [34]. An explanation for this behavior is the shape of the instability curves in the $\Theta_{0}-H a$ plane. By increasing the pinch ratio, different $m, n$ helical modes appear at a fixed $H a$, but they can disappear by increasing $\Theta_{0}$ to even higher values. This was investigated in [36].

In the next figures, 25 to 28 , we compare our different simulations with the ones performed by Shan et al.. We find quantitatively the same evolution of the average current density and the total dissipation rate $\left(\varepsilon_{T}=\lambda\left\langle j^{2}\right\rangle+v\left\langle\omega^{2}\right\rangle\right)$. These quantities are time averaged during the dynamical steady state, since the flow becomes chaotic, if the pinch ratio (or $E_{0}$ ) is large.

Some quantitative differences are observed in Figs. 25 and 26 for values around $E_{0}=0.6$. At that point both methods might not trigger exactly the same helical modes, since the electric field is imposed in a slightly different way. Both methods might therefore give results corresponding to different multi-mode states. When the pinch ratio is increased further, the deviations become smaller, as can be seen in Figs. 27 and 28.

This test-case allows us to validate the nonlinear MHD code with boundary conditions imposed on both the velocity and magnetic field. A linear analytical result is confirmed. The "multi-mode" and turbulent states that are observed in literature also appear in our simulations. Further simulations with varying Hartmann number to complete our picture of the nonlinear behavior of the system constitute an interesting perspective.

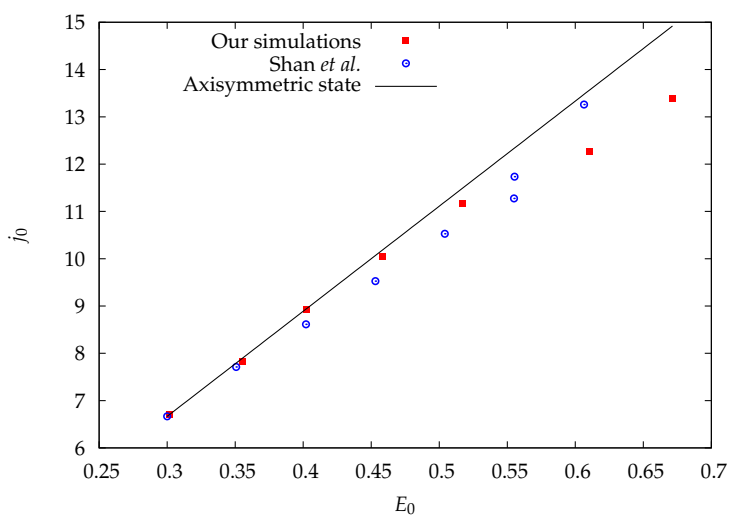

Figure 25: Zoom: average axial current as a function of the average electric field.

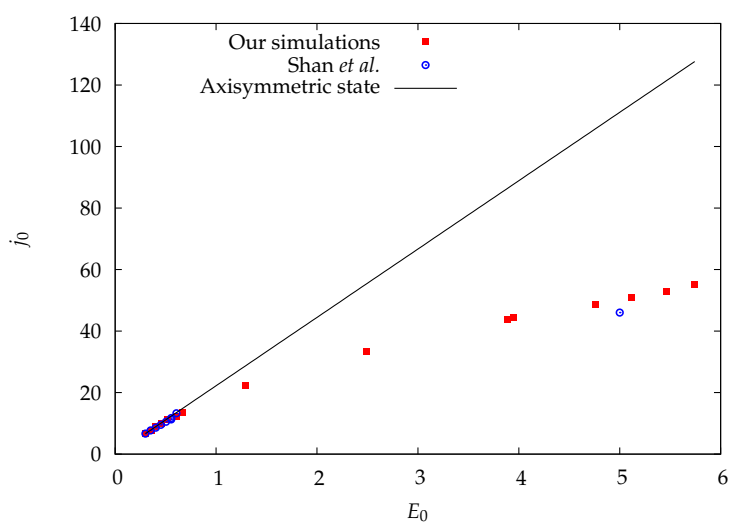

Figure 27: Average axial current as a function of the average electric field.

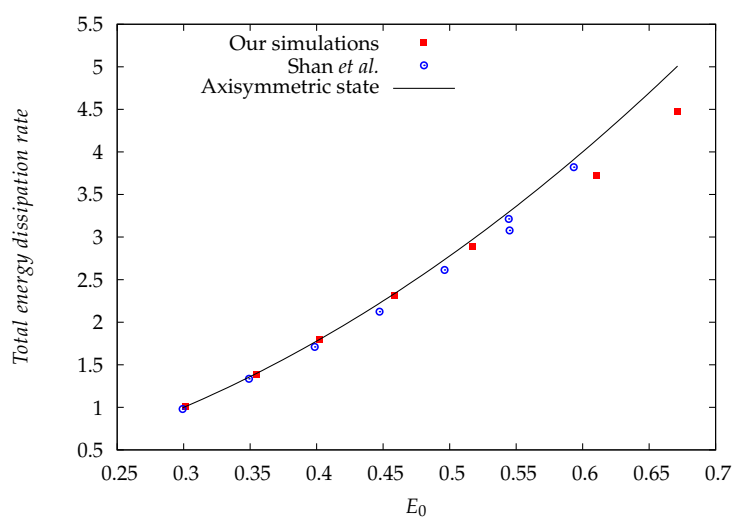

Figure 26: Zoom: total energy dissipation rate as a function of the average electric field.

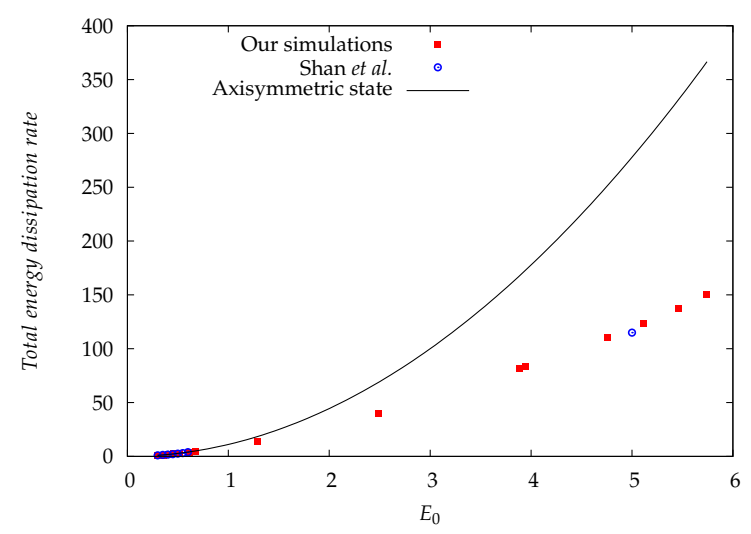

Figure 28: Total energy dissipation rate as a function of the average electric field. 


\section{Conclusion}

An extension and implementation of the penalization method into a pseudo-spectral Fourier code solving the MHD equations is presented. This penalization method, which allows the introduction of obstacles and walls in the computational domain, is implemented in different ways with respect to the numerical scheme and definition of the fields within the solid domains.

The numerical code is validated by comparison with several test-cases and theory. First in two dimensions the convergence of the results towards an analytical solution by decreasing the penalization parameter and increasing the resolution are shown. The method converges faster than second order if a regularization technique in the solid domain is applied which removes the discontinuities in the derivatives of the velocity and magnetic field at the solid-fluid boundary.

Then in three dimensions the first instability threshold of the hydrodynamic Taylor-Couette flow is found with good accuracy. Also the nonlinear saturation of this flow is compared and validated with the literature. For the MHD Taylor-Couette flow the current inability to impose non-homogeneous Neumann boundary conditions using the penalization method, makes the comparison of our numerical results with the literature difficult. A more appropriate test-case to validate the three-dimensional implementation of the penalization method to compute MHD flows is the flow induced by a helical magnetic field. This case is correctly reproduced. The linear threshold of the transition between an axisymmetric and a helical state is found. Also the evolution of the average axial current and the total dissipation rate as a function of the average electric field are compared with the literature and are in good agreement.

All these test-cases allow us to validate the numerical code to solve correctly the MHD equations in a confined domain. The limitation are the restricted magnetic boundary conditions that can be applied. Presently, the current density can be only be imposed indirectly via the magnetic field. A modified volume penalization method that allows the introduction of non-homogeneous Neumann boundary conditions is currently being developed.

\section{Acknowledgements}

We are very grateful to C. Jause-Labert, A. Delache, D. Kolomenskiy, F.S. Godeferd and S. Neffaa for the parallelization of the numerical code and for the help with the pseudo-spectral and volume penalization methods. We acknowledge financial support from the French Research Agency (ANR), project SiCoMHD, contract ANR-11-BLAN-045, as well as IDRIS for computing time, project i2012021664.

\section{Appendix A. Asymptotic analysis of the penalized momentum and induction equations}

This section follows the method used by Angot et al. [15] for the momentum equation in the hydrodynamic version. An asymptotic development of $\mathbf{u}$ and $\mathbf{B}$ with the small parameter $\eta$ is done first and gives

$$
\begin{aligned}
& \mathbf{u}=\mathbf{u}_{0}+\eta \mathbf{u}_{1}+\eta^{2} \mathbf{u}_{2}+\eta^{3} \mathbf{u}_{3}+\cdots, \\
& \mathbf{B}=\mathbf{B}_{0}+\eta \mathbf{B}_{1}+\eta^{2} \mathbf{B}_{2}+\eta^{3} \mathbf{B}_{3}+\cdots .
\end{aligned}
$$

Then these expressions are inserted into the governing equations (Eqs. (11) and (12))

$$
\begin{array}{r}
\partial_{t} \mathbf{u}=-\mathbf{u} \cdot \nabla \mathbf{u}-\nabla \Pi+\nu \nabla^{2} \mathbf{u}+\mathbf{j} \times \mathbf{B}-\frac{\chi}{\eta}\left(\mathbf{u}-\mathbf{u}_{\text {wall }}\right), \\
\partial_{t} \mathbf{B}=\nabla \times(\mathbf{u} \times \mathbf{B})+\lambda \nabla^{2} \mathbf{B}-\frac{\chi}{\eta}\left(\mathbf{B}-\mathbf{B}_{\text {wall }}\right)
\end{array}
$$

and we identify the terms of the same order in $\eta$.

order -1

$$
\left\{\begin{array}{l}
0=-\frac{\chi}{\eta}\left(\mathbf{u}_{0}-\mathbf{u}_{\text {wall }}\right) \\
0=-\frac{\chi}{\eta}\left(\mathbf{B}_{0}-\mathbf{B}_{\text {wall }}\right)
\end{array} \quad \Rightarrow \text { if } \chi=1\left\{\begin{array}{l}
\mathbf{u}_{0}=\mathbf{u}_{\text {wall }} \\
\mathbf{B}_{0}=\mathbf{B}_{\text {wall }}
\end{array}\right.\right.
$$


At this order, in the solid domain the fields are equal to the boundary conditions.

order 0

$$
\begin{array}{r}
\partial_{t} \mathbf{u}_{0}=-\mathbf{u}_{0} \cdot \nabla \mathbf{u}_{0}-\nabla \Pi_{0}+v \nabla^{2} \mathbf{u}_{0}+\nabla \times \mathbf{B}_{0} \times \mathbf{B}_{0}-\frac{\chi}{\eta} \mathbf{u}_{1}, \\
\partial_{t} \mathbf{B}_{0}=\nabla \times\left(\mathbf{u}_{0} \times \mathbf{B}_{0}\right)+\lambda \nabla^{2} \mathbf{B}_{0}-\frac{\chi}{\eta} \mathbf{B}_{1} .
\end{array}
$$

At this order, the Navier-Stokes and induction equations are found in the fluid $(\chi=0)$. In the solid domain $(\chi=1)$, the hypothesis that the fields of the order 0 are in a stationary state and homogeneous in space is made. In this case the equations reduce to

$$
\nabla \Pi_{0}=\frac{\chi}{\eta} \mathbf{u}_{1}, \quad 0=-\frac{\chi}{\eta} \mathbf{B}_{1}
$$

The first equation is Darcy's law. It is easily deduced that the penalization parameter $\eta$ must tend to zero for the velocity field to converge to the boundary condition value. The equation for the magnetic field remains not interpreted at the time.

order 1

$$
\begin{gathered}
\partial_{t} \mathbf{u}_{1}=-\mathbf{u}_{0} \cdot \nabla \mathbf{u}_{1}-\nabla \Pi_{1}+v \nabla^{2} \mathbf{u}_{1}+\nabla \times \mathbf{B}_{1} \times \mathbf{B}_{0}-\frac{\chi}{\eta} \mathbf{u}_{2}, \\
\partial_{t} \mathbf{B}_{1}=\nabla \times\left(\mathbf{u}_{1} \times \mathbf{B}_{0}\right)+\nabla \times\left(\mathbf{u}_{0} \times \mathbf{B}_{1}\right)+\lambda \nabla^{2} \mathbf{B}_{1}-\frac{\chi}{\eta} \mathbf{B}_{2} .
\end{gathered}
$$

Here the same hypothesis about the order 0 fields is made. There is no interpretation for this system, but we see that the system cannot be closed and we should truncate the series to do so.

\section{Appendix B. Corresponding explicit scheme of the semi-implicit penalization method}

Jause-Labert et al. [24] extended an implicit formulation for the time-integration scheme for Dirichlet conditions, originally proposed by Kolomenskiy and Schneider [17] for Burgers' equation, to relax the constraint on the choice of the time-step. Their approach is discussed in some detail in this section. We use the following form of the penalized Navier-Stokes equation:

$$
\partial_{t} u=u u-\partial_{x} P-\frac{\chi}{\eta} u
$$

in this equation $u_{\text {wall }}=0, P+u^{2} / 2 \rightarrow P$ and $u u$ represents the Lamb-vector. The viscous term can simply be added in the integral form, $u \exp \left(-v k^{2} t\right) \rightarrow u$. In Fourier space we can write

$$
\partial_{t} \hat{u}=P_{\perp}\left(\hat{u} u-\frac{1}{\eta} \widehat{\chi u}\right),
$$

with $P_{\perp}$ the Riesz projection-operator. The implicit treatment for the penalization term at the first order implies

$$
\frac{\hat{u}^{n+1}-\hat{u}^{n}}{\Delta t}=P_{\perp}\left(\hat{u} u^{n}-\frac{1}{\eta} \widehat{\chi u}^{n+1}\right),
$$

the fact that in the last term the $\hat{\chi}$ is convoluted with $\hat{u}^{n+1}$ makes it non-trivial to write the implicit formulation. The following formulation is proposed [24]:

$$
\hat{u}^{n+1}=P_{\perp} \mathcal{F}\left(\frac{u^{n}+\Delta t \mathcal{F}^{-1}\left[P_{\perp} \hat{u} u^{n}\right]}{1+\frac{\chi^{n+1}}{\eta} \Delta t}\right) .
$$

We know that the function $\chi$ takes only the values 1 and 0 depending if we are in the fluid or in the solid region

$$
\chi(x, t)= \begin{cases}0 & \text { for } x \in \Omega_{f}, \text { the fluid domain } \\ 1 & \text { for } x \in \Omega_{s}, \text { the solid domain. }\end{cases}
$$


An equivalent form of the Eq. (B.4) can be written as follows

$$
\hat{u}^{n+1}=P_{\perp} \mathcal{F}\left[\left(u^{n}+\Delta t \mathcal{F}^{-1}\left[P_{\perp} \hat{u u}^{n}\right]\right)\left(1-\chi^{n+1} \frac{\Delta t}{\eta+\Delta t}\right)\right] .
$$

We recover the Navier-Stokes equation in the fluid domain and the implicit penalized equation (B.4) in the solid region. Using the following relations

$$
\begin{aligned}
P_{\perp} P_{\perp} \hat{a} & =P_{\perp} \hat{a} \\
P_{\perp} \hat{u} & =\hat{u},
\end{aligned}
$$

the differential form of Eq. (B.6) can be written

$$
\partial_{t} \hat{u}=P_{\perp}\left(P_{\perp} \hat{u u}-\frac{\widehat{\chi u}}{\eta+\Delta t}-\frac{\Delta t}{\eta+\Delta t} \mathcal{F}\left\{\chi \mathcal{F}^{-1}\left[P_{\perp} \hat{u u}\right]\right\}\right) .
$$

Here we can identify two extreme cases. If $\Delta t<<\eta$ the equation (B.8) converges towards the equation (B.2), we recover the classical penalized Navier-Stokes equation. On the other hand if $\eta<<\Delta t$ we recover the following equation:

$$
\partial_{t} \hat{u}=P_{\perp}\left(\mathcal{F}\left\{(1-\chi) \mathcal{F}^{-1}\left[P_{\perp} \hat{u u}\right]\right\}-\frac{\widehat{\chi u}}{\Delta t}\right) .
$$

This equation is very close to the classical penalized Navier-Stokes equation. Here the time step $(\Delta t)$ replaces the penalization parameter $(\eta)$ and the nonlinear term vanishes in the solid region.

The semi-implicit penalization method is in this limit analogous to an explicit formulation. The difference is that, if the penalization parameter is small enough, the porosity of the solid walls is given by the value of the time step.

\section{References}

[1] P.A. Davidson. An introduction to magnetohydrodynamics. Cambridge Univ. Press, 2001

[2] C. Suplee. The plasma universe. Cambridge Univ. Press, 2009.

[3] S.C. Jardin. Review of implicit methods for the magnetohydrodynamic description of magnetically confined plasmas. J. Comp. Phys., 231(3):822-838, 2012.

[4] J.-L. Guermond, R. Laguerre, J. Léorat, and C. Nore. An interior penalty Galerkin method for the MHD equations in heterogeneous domains. J. Comp. Phys., 221(1):349-369, 2007.

[5] A.B. Iskakov, S. Descombes, and E. Dormy. An integro-differential formulation for magnetic induction in bounded domains: boundary element-finite volume method. J. Comp. Phys., 197(2):540-554, July 2004.

[6] K. Reuter, F. Jenko, C.B. Forest, and R.A. Bayliss. A parallel implementation of an MHD code for the simulation of mechanically driven, turbulent dynamos in spherical geometry. Comp. Phys. Comm., 179(4):245-249, 2008.

[7] G. Rüdiger and D. Shalybkov. Stability of axisymmetric Taylor-Couette flow in hydromagnetics. Phys. Rev. E, 66(1):016307, 2002.

[8] C. Gissinger, H. Ji, and J. Goodman. Instabilities in magnetized spherical Couette flow. Phys. Rev. E, 84(2):026308, 2011.

[9] A.P. Willis and C.F. Barenghi. Hydromagnetic Taylor-Couette flow: numerical formulation and comparison with experiment. J. Fluid Mech., 463(1):361-375, 2002.

[10] X. Shan, D.C. Montgomery, and H. Chen. Nonlinear magnetohydrodynamics by Galerkin-method computation. Phys. Rev. A, 44(10):6800, Nov 1991.

[11] P.D. Mininni and D.C. Montgomery. Magnetohydrodynamic activity inside a sphere. Phys. of Fluids, 18:116602, 2006.

[12] C.S. Peskin. The immersed boundary method. Acta Num., 11(1):479-517, 2002.

[13] E. Arquis and J.-P. Caltagirone. Sur les conditions hydrodynamiques au voisinage d'une interface milieu fluide-milieux poreux: application à la convection naturelle. C.R. Acad. Sci., 299:1-4, 1984.

[14] P. Angot and J.-P. Caltagirone. New graphical and computational architecture concept for numerical simulation on supercomputers. Proc. of 2nd World Cong. on Comp. Mech., 1(8):973-976, 1990.

[15] P. Angot, C.H. Bruneau, and P. Fabrie. A penalization method to take into account obstacles in viscous flows. Numerische Mathematik, 81:497-520, 1999.

[16] K. Schneider. Numerical simulation of the transient flow behaviour in chemical reactors using a penalisation method. Comput. $\mathcal{F}$ Fluids, 34(10):1223-1238, December 2005.

[17] D. Kolomenskiy and K. Schneider. A Fourier spectral method for the Navier-Stokes equations with volume penalization for moving solid obstacles. J. Comp. Phys., 228:5687-5709, May 2009.

[18] W.J.T. Bos, S. Neffaa, and K. Schneider. Rapid generation of angular momentum in bounded magnetized plasma. Phys. Rev. Lett., 101(23):235003, 2008. 
[19] S. Neffaa, W.J.T. Bos, and K. Schneider. The decay of magnetohydrodynamic turbulence in a confined domain. Phys. of Plasmas, 15:092304, 2008.

[20] W.J.T. Bos, S. Neffaa, and K. Schneider. Self-organization and symmetry-breaking in two-dimensional plasma turbulence. Phys. of Plasmas, 17:092302, 2010

[21] C. Canuto, M.Y. Hussaini, A. Quarteroni, and T.A. Zang. Spectral Methods in Fluid Dynamics. Springer, 1987.

[22] G. Carbou and P. Fabrie. Boundary layer for a penalization method for viscous incompressible flow. Adv. Differ. Equat., 8:1453, 2003.

[23] B. Kadoch, D. Kolomenskiy, P. Angot, and K. Schneider. A volume penalization method for incompressible flows and scalar advectiondiffusion with moving obstacles. J. Comp. Phys., 231:4365, 2012.

[24] C. Jause-Labert, F.S. Godeferd, and B. Favier. Numerical validation of the volume penalization method in three-dimensional pseudo-spectral simulations. Comput. $\mathcal{E}$ Fluids, in press, 2012.

[25] G.I. Taylor. Stability of a viscous liquid contained between two rotating cylinders. Phil. Trans. of the Royal Soc. of London, 223:289-343, 1923.

[26] S. Laizet and E. Lamballais. High-order compact schemes for incompressible flows: A simple and efficient method with quasi-spectral accuracy. J. Comp. Phys., 228(16):5989-6015, 2009.

[27] C. Guthmann and J.M. Rax. Physique des Plasmas. Dunod, 2005.

[28] P.D. Mininni, A.G. Pouquet, and D.C. Montgomery. Small-Scale Structures in Three-Dimensional Magnetohydrodynamic Turbulence. Phys. Rev. Lett., 97(24):1-4, December 2006.

[29] J.-L. Guermond, J. Léorat, and C. Nore. A new Finite Element Method for magneto-dynamical problems: two-dimensional results. Eur. J. of Mech. - B/Fluids, 22(6):555-579, December 2003.

[30] R. Laguerre, C. Nore, J. Léorat, and J.-L. Guermond. Induction effects in isolated axisymmetric conductors using a new finite element method. In Proc. of the Summer Program, page 1, 2004.

[31] J.-L. Guermond, R. Laguerre, J. Léorat, and C. Nore. Nonlinear magnetohydrodynamics in axisymmetric heterogeneous domains using a Fourier/finite element technique and an interior penalty method. J. Comp. Phys., 228(8):2739-2757, 2009.

[32] S. Chandrasekhar. Hydrodynamic and hydromagnetic stability. Dover Pubns, 1961.

[33] A.P. Willis and C.F. Barenghi. Hydromagnetic Taylor-Couette flow: wavy modes. J. Fluid Mech., 472:399-410, 2002.

[34] X. Shan, D.C. Montgomery, and H. Chen. Nonlinear magnetohydrodynamics by Galerkin-method computation. Phys. Rev. A, 44(10):6800$6818,1991$.

[35] D.C. Montgomery, L. Phillips, and M.L. Theobald. Helical, dissipative, magnetohydrodynamic states with flow. Phys. Rev. A, 40(3):1515, 1989.

[36] X. Shan and D.C. Montgomery. Global searches of Hartmann-number-dependent stability boundaries. Plasma Phys. Control. fusion, 35:1019, 1993. 